\title{
Energy-Spectral-Efficiency Analysis and Optimization of Heterogeneous Cellular Networks: A Large-Scale User-Behavior Perspective
}

\author{
Guogang Zhao, Sheng Chen, Fellow, IEEE, Liqiang Zhao, Member, IEEE and Lajos Hanzo, Fellow, IEEE
}

\begin{abstract}
Heterogeneous cellular networks (HCNs) are capable of meeting the explosive mobile-traffic demands. However, the conventional base station (BS) deployment strategy is unsuitable for supporting the often unpredictable non-uniform mobiletraffic demands, as governed by the large-scale user behavior (LUB). This results in the inefficient exploitation of the system's resources. In this paper, we develop an analytical framework for characterizing the achievable energy-spectral-efficiency (ESE) of HCNs, which explicitly quantifies the relationship between the network's ESE and the randomly time-varying LUBs as well as other network deployment parameters. Specifically, we model the quantitative impact of the geographical mobiletraffic intensity, the load migration factor, the users' required service rate and the per-tier $B S$ densities on the achievable ESE of network, while considering the area-spectral-efficiency requirements. Importantly, a closed-form ESE expression is derived, which enables us to explicitly analyze the properties of the network's ESE. Furthermore, the optimal LUB-aware BS deployment strategy is proposed for maximizing the ESE under specific outage constraints. Using numerical simulations, we verify the accuracy of the analytical ESE expression and quantify the impact of several relevant system parameters on the achievable ESE. Furthermore, we evaluate the achievable ESE performance of the network under diverse time-varying LUB scenarios. Our work therefore provides valuable insights for designing future ultra-dense HCNs.
\end{abstract}

Index Terms-Heterogeneous cellular networks, energyspectral-efficiency, large-scale-user-behaviors, base station deployment, mobile-traffic intensity, load migration factor, green communications and networks

\section{INTRODUCTION}

\section{A. Motivation}

Heterogeneous cellular networks (HCNs) are characterized by overlapping coverage areas supported both by the traditional macrocells and several different types of smallcells, whilst forming a large-scale irregularly and in many

This work was supported in part by National Natural Science Foundation of China (61771358), Intergovernmental International Cooperation on Science and Technology Innovation (2016YFE0123200), China Postdoctoral Science Foundation (2017M613074), Fundamental Research Funds for the Central Universities (JB170102), and the 111 Project (B08038). L. Hanzo would also like to acknowledge the financial support of the European Research Council's Advanced Fellow Grant Beam-Me-Up and the Royal Society's Wolfson Merit Award.

G. Zhao and L. Zhao (G.Zhao@soton.ac.uk, lqzhao@mail.xidian.edu.cn) are with the State Key Laboratory of Integrated Service Networks, Xidian University, Xi'an 710071, China.

S. Chen and L. Hanzo (sqc@ecs.soton.ac.uk, 1h@ecs.soton.ac.uk) are with the School of Electronics and Computer Science, University of Southampton, Southampton SO17 1BJ, UK. S. Chen is also with King Abdulaziz University, Jeddah 21589, Saudi Arabia.

IEEE Transactions in Vehicular Technology, 2018, accepted on 2/01/2018 places dense network architecture [1], [2]. Naturally, the areaspectral-efficiency (ASE) of the system is expected to be significantly improved owing to spatially reusing the available spectrum in a denser manner. Thus, the explosively increasing mobile traffic can still be seamlessly conveyed, whilst satisfying high quality-of-service (QoS) requirements. On the other hand, driven both by economical and environmental concerns, network designers have to pay increasing attention to power-efficient 'green radio' (GR) techniques [3]-[12] in order to curb the rapidly escalating energy consumption of wireless communication networks. Both the 3GPP Long Term Evolution (LTE) and LTE-Advanced standards have specified energy efficiency (EE) as an important system performance indicator in addition to the enhanced QoS guarantees and to the dramatic boost of service data rates [13]. Therefore, technologies that are capable of efficiently exploiting the limited energy resources within the given licensed spectrum are of paramount importance.

The energy consumed by base stations (BSs) dominates the network's total energy consumption, and it is severely influenced by the fluctuating and geographically non-uniform mobile traffic. In the traditional worst-case design, the underutilized BSs still consume substantial power simply for maintaining the cellular coverage [3]-[5]. This energy waste is serious, because again, the static power of a BS forms a predominant fraction of the total power consumption. Furthermore, the energy wastage may become more grave for larger-scale and ultra-dense networks [1], [2]. Therefore, it is critically important to appropriately capture and exploit the characteristics of the prevalent large-scale user behavior (LUB) for the sake of optimizing the network's spectral efficiency (SE) and/or EE subject to appropriate QoS guarantees in the context of large-scale irregularly and densely deployed multitier HCNs. Solving this challenging problem will result in energy-spectral efficient LUB-aware network design strategies. Unfortunately, it is inadequate to adopt the mobile-trafficaware BS deployment strategy [14] developed for homogeneous cellular systems to the more challenging multi-tier HCN scenarios. This is because in an $\mathrm{HCN}$, the LUBs include not only the geographically non-uniform mobile traffic, but also the spatial and temporal distribution of the mobile traffic amongst the tiers. Hence, in this paper, we will investigate sophisticated LUB-aware network-level designs that have the potential to save substantially more energy than the conventional methods that are unaware of the LUB. 


\section{B. Related Works}

A number of existing GR techniques focused on the energyefficient physical layer transceiver design and radio resource allocation optimization [8]-[12], which however did not take into account the network's traffic characteristics. Diverse BS energy saving methods relying on dynamic operations and on BS deployment strategies were investigated in [5]-[7], [15][17]. More specifically, several optimization problems were formulated for minimizing the energy consumption of a single cell or that of a certain network area, while considering the users' data rate requirements [6], [7], [15]-[17], where it was shown that the dynamic operation and BS deployment as well as the associated algorithms conceived for finding the optimal solutions impose a high computational complexity. Nevertheless, these schemes constitute case-specific studies and they provide locally optimized solutions, which may be further improved for employment in large-scale network-wide optimization. Additionally, the energy saving performance of the network deploying a small number of BSs was evaluated in [18]-[22], where a local cell area based on the ideal circular or hexagonal cell model was assumed. However, it requires further work to use the results of [18]-[22] for guiding the design and optimization of large-scale irregularly and densely deployed HCNs.

In modeling the distribution of large-scale irregularly and densely deployed BSs, the BSs' spatial positions are typically characterized using Poisson point processes (PPPs) [23], [24]. Several contributions further extended this PPP model to multi-tier cellular networks [25]-[27], where the concept of cell association or cell load amongst tiers becomes important. Recently, the authors of [28] focused their attention on the user mobility with human tendency and on the clustering behaviors in 5G small-cell networks. Based on these works, several energy efficient network deployment strategies were proposed in [29]-[32], where the authors aimed either for minimizing the area-power-consumption (APC) of the entire network or for maximizing the network's EE performance by choosing a suitable BS density unilaterally under usercentric performance constraints. To be more specific, in [29], [30], the network's APC was minimized by switching off as many of the BSs as possible. More explicitly, the BS density was minimized, subject to the per-tier coverage/outage performance constraints. The network's EE was improved with the aid of dormant BSs in [31], where the network's EE maximization problem was formulated as a function of the sleeping factors. However, the studies of [29]-[32] all assumed that each BS consumes a constant power, which implies that the network's APC is completely determined by the density of BSs. Consequently, the quantitative impacts of LUBs, including the mobile traffic intensity and the geographical mobile traffic distribution amongst tiers both on the BS's power consumption as well as on the network's SE and EE performance were not taken into account. Compared to the aforementioned contributions, the study [33] analyzed both the SE and EE of a large-scale cellular network. Our previous treatise [14] developed a mobile-traffic-aware BS deployment strategy for homogeneous networks, but it is difficult to adopt it for the analysis and optimization of large-scale multi-tier HCNs.

It is widely recognized that beneficially exploiting the LUBs in a large-scale multi-tier HCN to optimize the energyspectral-efficiency (ESE) is challenging, which has not been carried out in the existing literature.

\section{Our Contributions}

Against the above background, in this paper we propose an analytical ESE framework for large-scale irregularly and densely deployed $M$-tier HCNs, which facilitates the joint analysis of the impact of both the per-tier BS densities and the randomly time-varying LUBs, including the geographical mobile-traffic intensity and the load migration factor (LMF) ${ }^{1}$, as well as the average required service rate per tier. Based on this framework, we develop the optimal LUB-aware design strategies to achieve the maximum ESE for large-scale $M$ tier HCNs, while taking into account the outage performance constraints. Hence this paper explicitly answers the following questions: 1) What is the quantitative impact of both the LUB and the per-tier BS densities on the achievable network ESE? 2) Is it possible to obtain the optimal ESE solutions for largescale $M$-tier HCNs and how to exploit the optimal designs in practice? 3) How do the relevant system parameters influence the energy saving performance? Our main contributions are summarized as follows.

1) A new model for the BS's aggregate $D L$ transmit power: We model the quantitative relationship between the LUB and the BS's aggregate downlink (DL) transmit power in largescale irregularly and densely deployed $M$-tier HCNs, where the BS's aggregate DL transmit power is specified as the total DL power aggregated in each subband of a BS. In contrast to the related contributions [23]-[25], [29]-[32], where the BS's aggregate DL transmit power is assumed to be fixed and thus it is not influenced by the LUB, our model specifically characterizes the variations of the BS's aggregate DL transmit power caused by the geography-dependent mobile-traffic intensity and the LMF as well as the average required service rate per tier, the per-tier BS density and other network parameters. Furthermore, the modeling accuracy is verified with the aid of our simulation results.

2) A tractable network ESE analytical framework: An accurate and tractable network ESE expression is derived in closed-form based on the proposed BS aggregate DL transmit power model. This network ESE expression takes into account the per-tier BS densities, the LUB and several other relevant network parameters, and it is formulated as a function of the ratios of the BS densities to the geographical mobile-traffic intensities. The closed-form nature of this ESE expression makes the search of globally optimal solutions particularly tractable and efficient. Additionally, a closed-form equivalent condition is established for the LUB to maximize the ESE, which allows us to numerically determine the LUB-aware optimal BS densities and makes the LUB-aware optimal network design strategy achievable.

\footnotetext{
${ }^{1}$ The LMF reflects the mobile-traffic migration among the tiers, and it is similar to the bias factor of [23]-[27].
} 
3) System design insights and optimal strategy: According to the closed-form equivalent condition derived, we obtain several design guidelines for ultra-dense HCNs. In particular, we observe that the energy reduction achieved by our BS deployment strategy in a tier is partially offset by a denser BS deployment in other tiers. We propose an energy-efficient LUB-aware network design strategy for a $M$-tier HCN under outage probability constraints. Specifically, an optimal LUBaware BS deployment strategy is developed by choosing a suitable BS density for the tier considered, based on the current LUBs and the BS densities in other tiers.

The rest of this paper is structured as follows. In Section II, the system model is introduced. The ESE of a large-scale $M$-tier HCN is modeled in Section III. In Section IV, the achievable performance of the energy-spectral-efficient LUBaware network is analyzed, while the proposed optimal energy efficient LUB-aware network design strategy is provided in Section V. Our numerical simulation results are presented in Section VI, and our conclusions are offered in Section VII.

\section{System ModeL}

\section{A. M-tier HCN Characterization}

We consider a large-scale dense DL $M$-tier HCN, whose BSs are deployed irregularly. Specifically, the $M$ types of BSlocations are modeled by $M$ independent PPPs in the Euclidean plane $\mathbb{R}^{2}$, and the BSs of the $m$ th tier are collectively denoted by the set $\Psi_{m}$, which has the density $\lambda_{m}$, where $m \in\{1,2, \cdots, M\}$. Similarly, all the user equipments (UEs), denoted by the set $\Psi_{\mathrm{u}}=\left\{u_{i}\right\}$, are also spatially scattered according to an independent PPP having the density $\lambda_{\mathrm{u}}$. Fig. 1 depicts the topology of two-tier HCNs. Since the geographical mobile-traffic intensity is proportional to the users' spatial density $\lambda_{\mathrm{u}}$ given the average per-user data rate requirement, we will use $\lambda_{\mathrm{u}}$ to equivalently represent the mobile-traffic intensity. In contrast to a homogeneous network where the mobile-traffic intensity completely specifies the LUBs, the distribution of mobile traffic at each tier is also required for the $M$-tier HCN. We adopt the LMF to characterize the mobiletraffic distribution at every tier.

Specifically, let $z$ represent the coordinate position of a typical user. Furthermore, let $b_{m} \in \Psi_{m}$ be the nearest BS in the $m$ th tier to the typical user located at $z$. Then $z$ is associated with the $m$ th tier when the following condition is satisfied:

$$
\left\|z-b_{\widetilde{m}}\right\|^{-\alpha} \leq \mu_{m \leftarrow \widetilde{m}}\left\|z-b_{m}\right\|^{-\alpha}, \forall \widetilde{m} \neq m,
$$

where $\|\cdot\|$ denotes the Euclidean distance, and $\alpha$ is the pathloss exponent, while $\mu_{m \leftarrow \widetilde{m}}>0$ defines the LMF from tier $\widetilde{m}$ to tier $m$ having the property of $\mu_{m \leftarrow \widetilde{m}}=\frac{1}{\mu_{\widetilde{m} \leftarrow m}}$. Physically, the LMF is related to the mobile-traffic distribution at each tier [26], [27]. In general, increasing $\mu_{m \leftarrow \widetilde{m}}$ can cause an increased mobile-traffic at the edge of tier $\widetilde{m}$ to migrate to tier $m$, which is equivalent to extending the BS's coverage area in tier $m$, where the weighted Voronoi tessellation model of [23][27] can be adopted and the cell of $b_{m}$ is denoted by $V_{m}$. The effect of increasing $\mu_{m \leftarrow \widetilde{m}}$ to the topology of four-tier HCNs is illustrated in Fig. 2, where we have $m=$ micro, pico and femto tiers, while $\widetilde{m}=$ macro tier. The LMF is a long-term averaged quantity, where the fading is averaged out, and it is proportional to the ratio of the power-level received from BS in tier $m$ to the power level received from BS in tier $\widetilde{m}$ [26], [27]. For the two-tier HCN associated with $M=2$, let $m$ and $\widetilde{m}$ denote the macro tier and small tier, respectively. Since the power-level received from a macro BS is much higher than that from a small BS, the LMF $\mu_{m \leftarrow \widetilde{m}} \gg 1$ is true.

\section{B. Resource Assignment}

There are $N_{m}$ users located in $V_{m}$ and they are collectively denoted by the set $\varphi_{m}$. Additionally, unity frequency reuse is

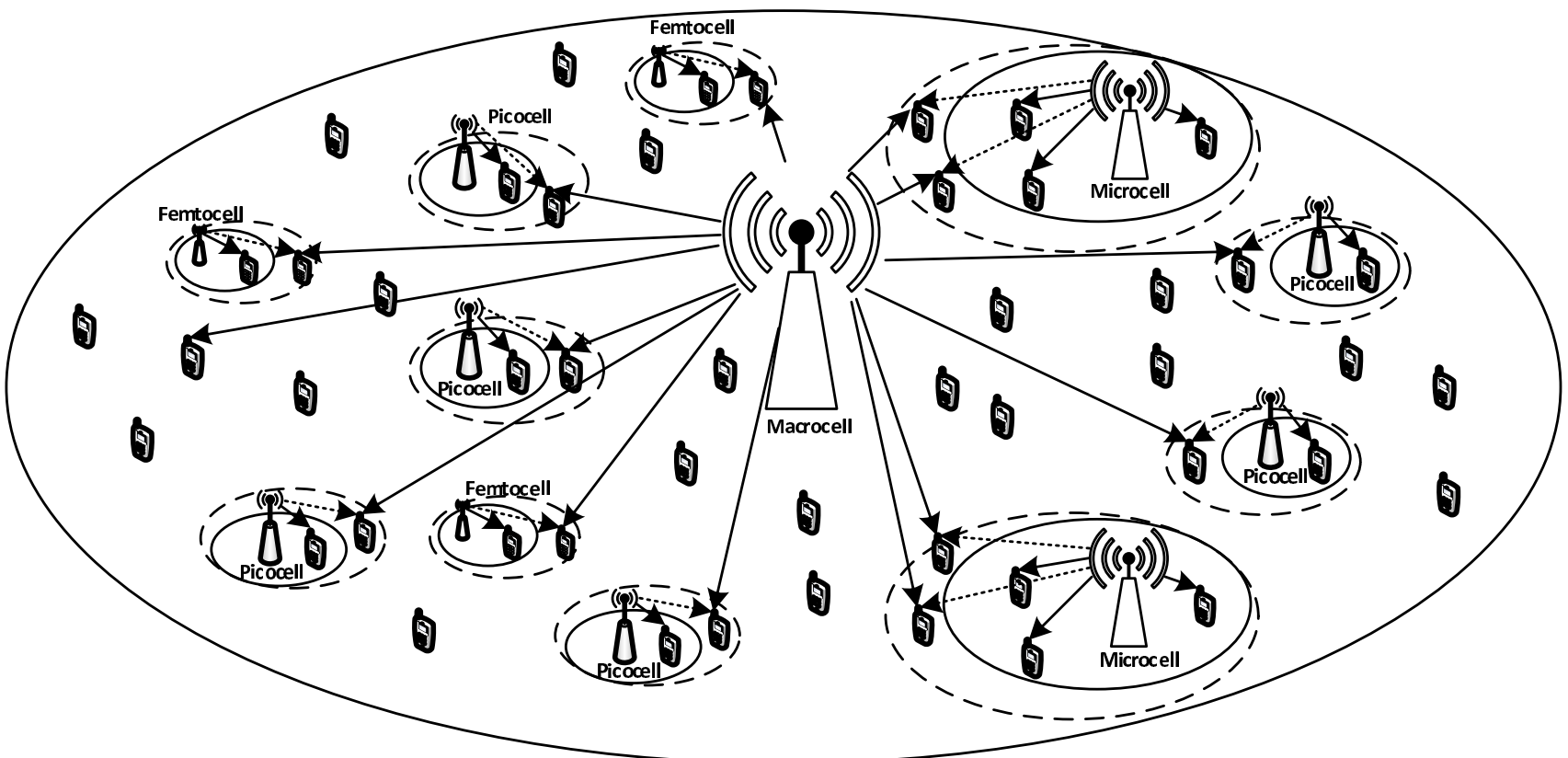

Fig 2: A four-tier HCN, where the solid circles around the small-cell BSs are their original coverage areas with the solid arrows as their original transmit signals, while the dashed circles represent their enlarged coverage areas with the dashed arrows as their new transmit signals when the LMFs $\mu_{m} \leftarrow$ mac are increased. 
employed for the cells, and the total bandwidth $B$ available to a cell $V_{m}$ is divided equally into $N_{m}$ subbands, with subband $B_{j, m}$ allocated to user $u_{j, m} \in \varphi_{m}$ for $1 \leq j \leq N_{m}$. In LTE systems, $B_{j, m}$ is constituted by a subset of the total set of subcarriers available. The random scheduling strategy is employed at the BS for achieving maximum fairness amongst users. The subband $B_{j, m}$ associated with user $u_{j, m}$ is then assigned an appropriate transmit power $P_{j, m}$ for meeting the service rate requirement $R_{j, m}$ of $u_{j, m}$ based on the long-term pathloss information. Each BS has a link request decision unit to manage its users' access to service. More specifically, the BS makes decisions regarding the access based on the current CSI and on the QoS requirements of the requesting users, who are only granted access when their QoS requirements can be satisfied. This resource assignment model characterizes the quantitative impact imposed by the various network parameters on each BS's aggregate DL transmit power, expressed as $P_{m}=$ $\sum_{j=1}^{N_{m}} P_{j, m}$, and $P_{m}$ is mainly influenced by the LUBs.

We consider both the large-scale pathloss and small-scale Rayleigh distributed fast fading on all desired and interfering links. The aggregate interference imposed on the UE located at $z$ is given by the total power received from all the BSs except its associated BS, i.e., from the BSs included in the set $\left(\bigcup_{\widetilde{m}=1}^{M} \Psi_{\widetilde{m}}\right) \backslash b_{m}$. Unless otherwise stated, the effect of noise is ignored, since the power of the aggregate interference at a given UE is far higher than the noise power in our interference-limited scenario. Without causing confusion, we also set $u_{j, m}=z$. In order to meet the rate requirement $R_{j, m}$, the DL transmit power $P_{j, m}$ of UE $u_{j, m}$ must satisfy

$$
R_{j, m}=B_{j, m} \log _{2}\left(1+\frac{P_{j, m} g_{j}^{m}}{\left\|z-b_{m}\right\|^{\alpha} I_{j, m}}\right),
$$

where $g_{j}^{m}$ is the power gain of the fast-fading channel spanning from BS $b_{m}$ to $z$, which is assumed to follow the independent exponential distribution with unity mean, i.e., $g_{j}^{m} \sim \exp (1)$, and $I_{j, m}$ denotes the corresponding received interference power. We focus our attention on the worst-case scenario, where the interfering BSs are transmitting at their maximum power $P_{\max }^{m}$. Hence,

$$
I_{j, m}=\sum_{\widetilde{m}=1}^{M} \sum_{b_{k} \in \Psi_{\widetilde{m}}, b_{k} \neq b_{m}}\left\|z-b_{k}\right\|^{-\alpha} \frac{P_{\max }^{\widetilde{m}}}{N_{m}} g_{j}^{k} .
$$

Because of adopting this worst-case interference model, the results obtained in this paper represent a lower-bound for the network's achievable performance.

\section{Performance Metric}

We define the network-level ESE metric for our large-scale $M$-tier HCN as follows:

$$
\eta_{\mathrm{ESE}}=\frac{\sum_{m=1}^{M} \mathrm{ASE}_{m}}{\sum_{m=1}^{M} \mathrm{APC}_{m}}[\mathrm{bit} / \mathrm{Hz} / \mathrm{Joule}],
$$

where $\mathrm{ASE}_{m}$ is the sum of the users' data rates [bit/s] per $\mathrm{m}^{2}$ per $\mathrm{Hz}$ in the $m$ th tier, and $\mathrm{APC}_{m}$ is the sum of the power consumption [Watt] per $\mathrm{m}^{2}$ in the $m$ th tier.

When deriving the ESE, we adopt the general power consumption model for each BS specified by the 3GPP standard
Table I: List of Main Notations.

\begin{tabular}{c|l}
\hline \hline Notation & Definition \\
\hline$\Psi_{m}$ & Set of BSs in tier $m$ \\
\hline$\lambda_{m}$ & Density of BSs in tier $m$ \\
\hline$\Psi_{\mathrm{u}}$ & Set of all UEs \\
\hline$\lambda_{\mathrm{u}}$ & Density of UEs \\
\hline$b_{m}$ & The nearest BS in tier $m$ to position $z$ \\
\hline$V_{m}$ & Cell centered at BS $b_{m}$ \\
\hline$\alpha$ & Pathloss exponent \\
\hline$\mu_{m \leftarrow \widetilde{m}}$ & LMF from tier $\widetilde{m}$ to tier $m$ \\
\hline$\varphi_{m}$ & Set of UEs associated with BS $b_{m}$ \\
\hline$N_{m}$ & Number of UEs in cell $V_{m}$ \\
\hline$B$ & Total bandwidth available to a cell \\
\hline$B_{j, m}$ & Subband allocated to UE $u_{j, m}$ \\
\hline$R_{j, m}$ & Required service data rate of UE $u_{j, m} \in \varphi_{m}$ \\
\hline$P_{j, m}$ & DL transmit power of UE $u_{j, m}$ \\
\hline$P_{m}$ & Aggregate DL transmit power of BS $b_{m}$ in tier $m$ \\
\hline$g_{j}^{m}$ & Fast-fading power gain from BS $b_{m}$ to UE $u_{j, m}$ \\
\hline$P_{\mathrm{max}}^{m}$ & maximum BS DL transmit power in tier $m$ \\
\hline$\eta_{\mathrm{ESE}}$ & Network-level energy-spectral efficiency \\
\hline$A_{m}$ & Coverage area of cell $V_{m}$ \\
\hline$\beta_{m}$ & tier $m$ BS power amplifier efficiency \\
\hline$P_{\mathrm{OM}}^{m}$ & Each BS's static operation power in tier $m$ \\
\hline$R_{m}$ & Average required service data rate (per UE) in tier $m$ \\
\hline$Q_{m}$ & Outage probability in tier $m$ \\
\hline$\varepsilon_{\mathrm{out}}$ & Outage threshold \\
\hline \hline &
\end{tabular}

[4] and use $P_{\text {tot }}^{m}$ to denote the total BS power consumption in tier $m$. Additionally, a user is regarded to suffer from outage, if its service data rate cannot be guaranteed under the maximum BS transmit power available to the subband occupied by this user. Since our main goal is the network's ESE-level evaluation, the users' outage probabilities are treated as the constraints.

For clarity, the main notations used in this paper are summarized in Table I.

\section{NETWORK-LEVEL ESE FORMULATION IN LARGE-SCALE $M$-TIER HCNS}

For the large-scale $M$-tier HCN, we characterize the average DL transmit power for a typical user and the average aggregate DL transmit power in a typical cell as well as derive a closedform network-level ESE expression. In particular, we quantify the impact of LUB, including the geographical mobile-traffic intensity and LMFs, and the spatially averaged service data rate requirement.

\section{A. Average DL Transmit Power for a Typical User}

Proposition 1. In a large-scale $M$-tier HCN associated with irregularly and densely deployed BSs, given the BS densities $\lambda_{m}$, the mobile-traffic intensity $\lambda_{\mathrm{u}}$ and the LMFs $\mu_{m \leftarrow \widetilde{m}}$, the average $D L$ transmit power required for user $u_{j, m}$ having the service data rate of $R_{j, m}$ is given by

$$
E\left[P_{j, m}\right]=\frac{\lambda_{m}\left(2^{R_{j, m} / B_{j, m}}-1\right)}{(\alpha-2) \lambda_{\mathrm{u}}} \sum_{\widetilde{m}=1}^{M} \frac{\lambda_{\widetilde{m}} P_{\max }^{\widetilde{m}} \mu_{m \leftarrow \widetilde{m}}^{(\alpha-2) / \alpha}}{\lambda_{m}},
$$

where E[.] denotes the expectation operator, and the pathloss exponent is $\alpha>2$.

Proof. See Appendix A.

The impact of the mobile-traffic intensity $\lambda_{\mathrm{u}}$ and the BS densities $\lambda_{m}$ on the average DL transmit power required by 
user $u_{j, m}$ is multifold. From the first fractional expression on the right-hand side of (5), we can see that increasing $\lambda_{m}$, which indicates having denser interfering BSs from tier $m$, is beneficial for increasing $E\left[P_{j, m}\right]$. However, a larger $\lambda_{m}$ increases $B_{j, m}$ since each cell has less users, which in turn decreases $E\left[P_{j, m}\right]$. Since this reduction is exponential, $E\left[P_{j, m}\right]$ decreases as $\lambda_{m}$ increases. On the other hand, $E\left[P_{j, m}\right]$ includes the factor of $\frac{1}{\lambda_{\mathrm{u}}}$, and hence it decreases as $\lambda_{u}$ increases. But increasing $\lambda_{\mathrm{u}}$ will results in less subbands $B_{j, m}$ being allocated for $u_{j, m}$, which in turn increases $E\left[P_{j, m}\right]$ exponentially. Therefore, increasing $\lambda_{\mathrm{u}}$ will increase $E\left[P_{j, m}\right]$ in general. Furthermore, increasing the LMF $\mu_{m \leftarrow \widetilde{m}}$ implicitly also reduces $B_{j, m}$, because more users will migrate from tier $\widetilde{m}$ to $m$, which results in a higher mobile traffic in tier $m$. Note that from (5), by setting $M=1$ and $\mu_{m \leftarrow \widetilde{m}}=1$, the average DL transmit power required for a typical UE in the homogeneous cellular network can be obtained [14].

\section{B. Average Aggregate DL Transmit Power in a Typical Cell}

Proposition 2. In a large-scale $M$-tier HCN with irregularly and densely deployed BSs, given the BS densities $\lambda_{m}$, the mobile-traffic intensity $\lambda_{\mathrm{u}}$ and the LMFs $\mu_{m \leftarrow \widetilde{m}}$, upon assuming that all the users in tier $m$ have an identical required service data rate of $R_{m}$, the averaged aggregate $D L$ transmit power of $B S b_{m}$ is given by

$$
\begin{aligned}
E\left[P_{m}\right]= & \left(K\left(\sum_{\widetilde{m}=1}^{M} \lambda_{\widetilde{m}} \mu_{m \leftarrow \widetilde{m}}^{-2 / \alpha}\right)-\left(2^{R_{m} / B}-1\right) \lambda_{\mathrm{u}}\right)^{-K} \\
& \times \frac{P_{\max }^{m}}{(\alpha-2)}\left(\sum_{\widetilde{m}=1}^{M} K \lambda_{\widetilde{m}} \mu_{m \leftarrow \widetilde{m}}^{-2 / \alpha}\right)^{K}-\frac{P_{\max }^{m}}{(\alpha-2)},
\end{aligned}
$$

where $K=3.57$.

Proof. See Appendix B.

\section{Network-Level ESE Formulation}

We are now ready to complete the network-level ESE metric (4) for a large-scale $M$-tier HCN. By using $R_{m}$ to represent the users' spatially averaged required service data rate in tier $m$, the ASE can be expressed as

$$
\mathrm{ASE}=\sum_{m=1}^{M} \frac{1}{B} \frac{\lambda_{\mathrm{u}} R_{m}}{\sum_{\widetilde{m}=1}^{M} \frac{\lambda_{\widetilde{m}} \mu_{m \leftarrow \widetilde{m}}^{-2 / \alpha}}{\lambda_{m}}}\left[\mathrm{bit} / \mathrm{s} / \mathrm{Hz} / \mathrm{m}^{2}\right],
$$

where $\lambda_{\mathrm{u}}$ has the unit of $\mathrm{UE} / \mathrm{m}^{2}$, and

$$
\frac{1}{\sum_{\widetilde{m}=1}^{M} \frac{\lambda_{\widetilde{m}}}{\lambda_{m}} \mu_{m \leftarrow \widetilde{m}}^{-2 / \alpha}}=\frac{1}{\chi_{m}}
$$

represents the number of BSs associated with tier $m$ [26], [27]. The network's APC on the other hand can be expressed as

$$
\mathrm{APC}=\sum_{m=1}^{M} \lambda_{m}\left(\beta_{m} E\left[P_{m}\right]+P_{\mathrm{OM}}^{m}\right)\left[\text { Joule } / \mathrm{s} / \mathrm{m}^{2}\right]
$$

where $\lambda_{m}\left(\beta_{m} E\left[P_{m}\right]+P_{\mathrm{OM}}^{m}\right)$ represents the BS power consumption per $\mathrm{m}^{2}$ in tier $m, \beta_{m}$ is the $m$ th tier BS power amplifier efficiency, and $P_{\mathrm{OM}}^{\mathrm{m}}$ represents each tier- $m$ BS's static operational power consumption, including the power consumed by baseband processing, battery backup, BS cooling, and so on. Thus,

$$
\eta_{\mathrm{ESE}}=\frac{\sum_{m=1}^{M} \frac{\lambda_{\mathrm{u}} \lambda_{m} R_{m}}{B \sum_{\bar{m}=1}^{M} \lambda_{\widetilde{m}} \mu_{m \leftarrow \widetilde{m}}^{-2 / \alpha}}}{\sum_{m=1}^{M} \lambda_{m}\left(\beta_{m} E\left[P_{m}\right]+P_{\mathrm{OM}}^{m}\right)}[\mathrm{bits} / \mathrm{Hz} / \mathrm{Joule}]
$$

Noting Proposition 2, the following proposition is obvious.

Proposition 3. In a large-scale $M$-tier $H C N$ with irregularly and densely deployed BSs having the BS density $\lambda_{m}$ in tier $m$, the mobile-traffic intensity $\lambda_{\mathrm{u}}$ and the $L M F \mu_{m \leftarrow \widetilde{m}}$, upon assuming that all the users in tier $m$ have an identical required service data rate of $R_{m}$, the closed-form network-level ESE expression is given by (11) at the bottom of this page.

Additionally, in the special case of $M=2$, we have the following corollary.

Corollary 1. Consider the large-scale two-tier HCN, where the density of macro BSs is $\lambda_{\text {mac }}$, the density of small BSs is $\lambda_{\text {mic }}$, the mobile-traffic intensity is $\lambda_{\mathrm{u}}$, and the $L M F$ is $\mu_{\text {mac } \leftarrow \text { mic }}=\mu$, while the average required service data rates in the macro-BS tier and small-BS tier are $R_{\mathrm{mac}}$ and $R_{\mathrm{mic}}$, respectively. The closed-form network-level ESE expression for this two-tier HCN is given by

$$
\eta_{\mathrm{ESE}}=\frac{R_{\mathrm{mac}} / \chi_{\mathrm{mac}}+R_{\mathrm{mic}} / \chi_{\mathrm{mic}}}{B\left(U_{\mathrm{mac}}+U_{\mathrm{mic}}\right)}[\mathrm{bit} / \mathrm{Hz} / \mathrm{Joule}],
$$

$$
\eta_{\mathrm{ESE}}=\frac{\sum_{m=1}^{M} \frac{R_{m} \lambda_{m}}{B \sum_{\bar{m}=1}^{M} \lambda_{\widetilde{m}} \mu_{m \leftarrow \widetilde{m}}^{-2 / \alpha}}}{\sum_{m=1}^{M}\left(\frac{P_{\max }^{m} \beta_{m} \lambda_{m}}{(\alpha-2) \lambda_{\mathrm{u}}\left(1-\left(2^{R_{m} / B}-1\right) \lambda_{\mathrm{u}} /\left(K \sum_{\tilde{m}=1}^{M} \lambda_{\widetilde{m}} \mu_{m \leftarrow \widetilde{m}}^{-2 / \alpha}\right)\right)^{K}}-\frac{\lambda_{m} \beta_{m} P_{\max }^{m}}{\lambda_{\mathrm{u}}(\alpha-2)}+\frac{\lambda_{m} P_{\mathrm{OM}}^{m}}{\lambda_{\mathrm{u}}}\right)}[\mathrm{bits} / \text { Hz } / \text { Joule]. }
$$


where

$$
\begin{aligned}
\chi_{\mathrm{mac}}= & 1+\frac{\lambda_{\mathrm{mic}}}{\lambda_{\mathrm{mac}}} \mu^{-\frac{2}{\alpha}}, \\
\chi_{\mathrm{mic}}= & 1+\frac{\lambda_{\mathrm{mac}}}{\lambda_{\mathrm{mic}}} \mu^{\frac{2}{\alpha}}, \\
U_{\mathrm{mac}}= & \frac{P_{\mathrm{max}}^{\mathrm{mac}} \beta_{\mathrm{mac}} \lambda_{\mathrm{mac}}}{(\alpha-2) \lambda_{\mathrm{u}}\left(1-\frac{\left(2^{R_{\mathrm{mac}} / B}-1\right) \lambda_{\mathrm{u}}}{K \lambda_{\mathrm{mac}} \chi_{\mathrm{mac}}}\right)^{K}} \\
& -\frac{\lambda_{\mathrm{mac}} \beta_{\mathrm{mac}} P_{\max }^{\operatorname{mac}}}{\lambda_{\mathrm{u}}(\alpha-2)}+\frac{\lambda_{\mathrm{mac}} P_{\mathrm{OM}}^{\mathrm{mac}}}{\lambda_{\mathrm{u}}}, \\
U_{\mathrm{mic}}= & \frac{(\alpha-2) \lambda_{\mathrm{u}}\left(1-\frac{\left(2^{R_{\mathrm{mic}} / B}-1\right) \lambda_{\mathrm{u}}}{K \lambda_{\mathrm{mic}} \chi_{\mathrm{mic}}} \beta_{\mathrm{mic}} \lambda_{\mathrm{mic}}\right.}{\lambda_{\mathrm{mic}}} \\
& -\frac{\lambda_{\mathrm{mic}} \beta_{\mathrm{mic}} P_{\mathrm{max}}^{\operatorname{mic}}}{\lambda_{\mathrm{u}}(\alpha-2)}+\frac{\lambda_{\mathrm{mic}} P_{\mathrm{OM}}^{\mathrm{mic}}}{\lambda_{\mathrm{u}}} .
\end{aligned}
$$

\section{LUBS-AwARE PERFormanCE ANALYSIS AND OPTIMIZATION IN LARGE-SCALE $M$-TIER HCNS}

In this section, the properties of the network-level ESE for a large-scale $M$-tier HCN are explicitly analyzed. First, the optimal LUB-aware BS densities maximizing the network's ESE are derived in the following proposition.

Proposition 4. In a large-scale $M$-tier HCN having the BS density $\lambda_{m}$ in tier $m$, the mobile-traffic intensity $\lambda_{\mathrm{u}}$ and the $L M F \mu_{m \leftarrow \tilde{m}}$, where all the users in tier $m$ have an identical required service data rate of $R_{m}$, there exists a unique optimal LUB-aware BS density in tier $m$, denoted by $\lambda_{m}^{\star}$, which maximizes the network's ESE $\eta_{\mathrm{ESE}}$. Furthermore, $\lambda_{m}^{\star}$ can be numerically obtained from

$$
\frac{1-(1+K) \Upsilon_{m}}{\left(1-\Upsilon_{m}\right)^{K+1}}+\sum_{\widetilde{m} \neq m} \mu_{m \leftarrow \widetilde{m}}^{\frac{2-\alpha}{\alpha}} \frac{1-(1+K) \Upsilon_{\widetilde{m}}}{\left(1-\Upsilon_{\widetilde{m}}\right)^{K+1}}=\gamma_{m}^{(M)},
$$

where

$$
\begin{gathered}
\Upsilon_{m}=\frac{\left(2^{R_{m} / B}-1\right) \lambda_{\mathrm{u}}}{K\left(\lambda_{m}^{\star}+\sum_{\widetilde{m} \neq m} \lambda_{\widetilde{m}} \mu_{m \leftarrow \widetilde{m}}^{-\frac{2}{\alpha}}\right)}, \\
\Upsilon_{\widetilde{m}}=\frac{\left(2^{R_{\tilde{m}} / B}-1\right) \lambda_{\mathrm{u}}}{K\left(\lambda_{\widetilde{m}}+\sum_{m^{\prime} \neq m, m^{\prime} \neq \widetilde{m}} \lambda_{m^{\prime}} \mu_{\widetilde{m} \leftarrow m^{\prime}}^{-\frac{2}{\alpha}}+\lambda_{m}^{\star} \mu_{\widetilde{m} \leftarrow m}^{-\frac{2}{\alpha}}\right)}, \\
\gamma_{m}^{(M)}=\left(1+\sum_{\widetilde{m} \neq m} \mu_{m \leftarrow \widetilde{m}}^{\frac{2-\alpha}{\alpha}}\right)-\frac{P_{\mathrm{OM}}^{m}}{P_{\max }^{m}}(\alpha-2) .
\end{gathered}
$$

Proof. See Appendix C.

In the special case of $M=2$, i.e., a large-scale macro-small two-tier HCN, we have the following corollary.

Corollary 2. In a large-scale two-tier HCN with irregularly and densely deployed macro BSs in tier mac and small BSs in tier mic, the unique optimal LUBs-aware BS density $\lambda_{m}^{\star}$ can be numerically obtained from

$$
\frac{1-(1+K) \bar{\Upsilon}_{m}}{\left(1-\bar{\Upsilon}_{m}\right)^{K+1}}+\mu_{m \leftarrow \widetilde{m}}^{\frac{2-\alpha}{\alpha}} \frac{1-(1+K) \bar{\Upsilon}_{\widetilde{m}}}{\left(1-\bar{\Upsilon}_{\widetilde{m}}\right)^{K+1}}=\gamma_{m},
$$

where $m=\operatorname{mac}$ and $\tilde{m}=\operatorname{mic}$ or $m=\operatorname{mic}$ and $\widetilde{m}=\operatorname{mac}$,

$$
\begin{aligned}
\bar{\Upsilon}_{m} & =\frac{\left(2^{R_{m} / B}-1\right) \lambda_{\mathrm{u}}}{K\left(\lambda_{m}^{\star}+\lambda_{\widetilde{m}} \mu_{m \leftarrow \widetilde{m}}^{-\frac{2}{\alpha}}\right)}, \\
\bar{\Upsilon}_{\widetilde{m}} & =\frac{\left(2^{R_{\widetilde{m}} / B}-1\right) \lambda_{\mathrm{u}}}{K\left(\lambda_{\widetilde{m}}+\lambda_{m}^{\star} \mu_{m \leftarrow \widetilde{m}}^{\frac{2}{\alpha}}\right)}, \\
\gamma_{m} & =\left(1+\mu_{m \leftarrow \widetilde{m}}^{\frac{2-\alpha}{\alpha}}\right)-\frac{P_{\mathrm{OM}}^{m}}{P_{\max }^{m}}(\alpha-2) .
\end{aligned}
$$

From Corollary 2, we have the following corollary.

Corollary 3. Consider the large-scale two-tier HCN with irregularly and densely deployed macro BSs in tier mac and small BSs in tier mic. Given two BS densities $\lambda_{\widetilde{m}, 1} \neq \lambda_{\widetilde{m}, 2}$ in tier $\widetilde{m}$, we have a pair of corresponding optimal BS densities $\lambda_{m, 1}^{\star}$ and $\lambda_{m, 2}^{\star}$ in tier $m$, which satisfy

$$
\left(\lambda_{m, 1}^{\star}-\lambda_{m, 2}^{\star}\right)\left(\lambda_{\widetilde{m}, 1}-\lambda_{\widetilde{m}, 2}\right)<0
$$

where $m=\operatorname{mac}$ and $\tilde{m}=\operatorname{mic}$ or $m=\operatorname{mic}$ and $\tilde{m}=$ mac.

Proof. See Appendix D.

Corollary 3 implies that when more BSs are deployed in tier $\widetilde{m}$, i.e., when increasing $\lambda_{\widetilde{m}}$, the optimal BS density $\lambda_{m}^{\star}$ in tier $m$ has to be reduced, if the network is expected to operate at a near-optimal ESE. This is consistent with the generally accepted insight that deploying denser macro BSs will degrade the performance of small BSs due to the increased cross-tier interference imposed on small cells, and vice versa.

Proposition 5. Consider the large-scale $M$-tier HCN with irregularly and densely deployed BSs having the BS density $\lambda_{m}$ in tier $m$ and the $L M F \mu_{m \leftarrow \widetilde{m}}$, where all the users in tier $m$ have an identical required service rate of $R_{m}$. There exists a unique optimal mobile-traffic intensity for maximizing the network ESE, denoted as $\lambda_{\mathrm{u}}^{\star}$, which can be numerically obtained from

$$
\begin{aligned}
& \sum_{m=1}^{M}\left(\frac{K P_{\max }^{m} \beta_{m} \lambda_{m} \lambda_{\mathrm{u}}^{\star} S_{m}}{\left(1-S_{m} \lambda_{\mathrm{u}}^{\star}\right)^{K+1}}-\frac{P_{\max }^{m} \beta_{m} \lambda_{m}}{\left(1-S_{m} \lambda_{\mathrm{u}}^{\star}\right)^{K}}\right. \\
& \left.\quad+\lambda_{m} \beta_{m} P_{\max }^{m}-\lambda_{m} P_{\mathrm{OM}}^{m}(\alpha-2)\right)=0
\end{aligned}
$$

where

$$
S_{m}=\frac{\left(2^{R_{m} / B}-1\right)}{\left(K \sum_{\widetilde{m}=1}^{M} \lambda_{\widetilde{m}} \mu_{m \leftarrow \widetilde{m}}^{-2 / \alpha}\right)} .
$$

Proof. See Appendix E. 


\section{Optimal ENERGY EFFICIENT LUBS-AWARE NeTwork Design StRATEGY}

In this section, we commence by presenting our framework conceived for designing the optimal energy efficient LUBaware $M$-tier HCN. However, our main goal is to derive an energy efficient LUBs-aware design strategy for a large-scale two-tier HCN with irregularly and densely deployed macro BSs in tier mac and small BSs in tier mic.

\section{A. Optimal Energy Efficient LUBs-Aware Network Design Strategy for $M$-tier HCNs}

The following proposition presents the outage probability of a typical user associated with a tier- $m$ BS, which is defined as the probability that a typical user's data rate requirement cannot be met even at the maximum DL transmit power.

Proposition 6. In a large-scale $M$-tier $H C N$ with irregularly and densely deployed BSs where the BS-density is $\lambda_{m}$ in tier $m$ with the $L M F \mu_{m \leftarrow \widetilde{m}}$ and the mobile-traffic intensity is $\lambda_{\mathrm{u}}$, the outage probability of a typical user $u_{j, m}$ associated with a tier-m $B S b_{m}$ that requires a service data rate of $R_{j, m}$ is given by

$$
\begin{aligned}
Q_{j, m}= & \frac{\lambda_{\mathrm{u}}}{N_{m} \sum_{\widetilde{m}=1}^{M} \mu_{m \leftarrow \widetilde{m}}^{-\frac{2}{\alpha}} \lambda_{\widetilde{m}} \rho_{j, m} \exp \left(\frac{N_{m}}{\lambda_{\mathrm{u}}} \sum_{\tilde{m}=1}^{M} \mu_{m \leftarrow \widetilde{m}}^{-\frac{2}{\alpha}} \lambda_{\widetilde{m}} \rho_{j, m}\right)} \\
& +1-\frac{\lambda_{\mathrm{u}}}{N_{m} \sum_{\widetilde{m}=1}^{M} \mu_{m \leftarrow \widetilde{m}}^{-\frac{2}{\alpha}} \lambda_{\widetilde{m}} \rho_{j, m}}
\end{aligned}
$$

where $N_{m}$ is the number of UEs associated with BS $b_{m}$, whose probability mass function (PMF) can be found in Appendix B, and

$$
\rho_{j, m}=T_{j, m}^{2 / \alpha} \int_{T_{j, m}^{-2 / \alpha}}^{+\infty} \frac{1}{1+u^{\alpha / 2}} d u
$$

with $T_{j, m}=2^{R_{j, m} / B_{j, m}}-1$.

Proof. See Appendix F.

In $Q_{j, m}$, by replacing $N_{m}$ with $E\left[N_{m}\right]$ of (52) in Appendix $\mathrm{B}$ and by replacing $R_{j, m}$ with the average service data rate $R_{m}$, we obtain the outage probability of the $m$ th tier as

$$
Q_{m}=1+\frac{1}{\rho_{m} \exp \left(\rho_{m}\right)}-\frac{1}{\rho_{m}},
$$

in which

$$
\rho_{m}=T_{m}^{2 / \alpha} \int_{T_{m}^{-2 / \alpha}}^{+\infty} \frac{1}{1+u^{\alpha / 2}} d u
$$

with

$$
T_{m}=2^{\left(R_{m} \lambda_{\mathrm{u}}\right) /\left(B \sum_{\tilde{m}=1}^{M} \mu_{m \leftarrow \widetilde{m}}^{-2 / \alpha} \lambda_{\widetilde{m}}\right)}-1 .
$$

Denote $\boldsymbol{\lambda}=\left[\begin{array}{llll}\lambda_{1} & \lambda_{2} & \cdots & \lambda_{M}\end{array}\right]^{\mathrm{T}}$. Clearly, $Q_{m}$ is a function of $\boldsymbol{\lambda}$ and $\lambda_{\mathrm{u}}$, and is therefore denoted as $Q_{m}\left(\boldsymbol{\lambda}, \lambda_{\mathrm{u}}\right)$.
The problem of finding the optimal LUB-aware energy efficient BS deployment for the $M$-tier $\mathrm{HCN}$ under the outage constraint in each tier can be formulated as

$$
\begin{array}{cl}
\max _{\boldsymbol{\lambda}} & \eta_{\mathrm{ESE}}, \\
\text { s.t. } & Q_{m}\left(\boldsymbol{\lambda}, \lambda_{\mathrm{u}}\right) \leq \varepsilon_{\text {out }}^{m}, 1 \leq m \leq M,
\end{array}
$$

where $0<\varepsilon_{\text {out }}^{m}<1$ is the outage probability threshold in the $m$ th tier and $\eta_{\mathrm{ESE}}$ is given by (11).

The optimization problem (33) is complex. Moreover, its practical application is somewhat limited, since it is unlikely in practice that a $M$-tier $\mathrm{HCN}$ is designed and deployed from the beginning. A most likely practical senario is that when a homogeneous macro network can no longer meet the service requirements, a micro tier is introduced. Therefore, the task becomes one of optimizing the small BS deployment to achieve the optimal ESE operating status of the two-tier HCN. We will study this more tractable and practical two-tier $\mathrm{HCN}$ senario, whose topology is illustrated in Fig. 1.

\section{B. Optimal Energy Efficient LUBs-Aware Network Design Strategy for Two-tier HCNs}

We now focus our attention on large-scale two-tier HCNs and our task is to find the optimal LUB-aware small BS density under the relevant outage constraint in the macro tier associated with the given macro BS density $\lambda_{\text {mac }}$ and user intensity $\lambda_{\mathrm{u}}$, which will be denoted as $\lambda_{\text {mic }}^{\text {out }}$. The optimization problem associated with this optimal LUB-aware small BS deployment design for the two-tier $\mathrm{HCN}$ is formally formulated as

$$
\begin{array}{cl}
\underset{\lambda_{\text {min }}}{\max } & \eta_{\mathrm{ESE}}, \\
\text { s.t. } & Q_{\text {mac }}\left(\lambda_{\text {mic }} ; \lambda_{\text {mac }}, \lambda_{\mathrm{u}}\right) \leq \varepsilon_{\text {out }},
\end{array}
$$

where $0<\varepsilon_{\text {out }}<1$ is the outage probability threshold in tier mac and $\eta_{\mathrm{ESE}}$ is given by (12). First, we explain why only the macro-tier outage constraint has to be considered. A smallcell user not only gets more bandwidth, since the number of UEs in a small cell is relatively small in comparison with a macro cell, but also requires less transmit power to meet its rate requirement, since the distance involved is much smaller. Therefore, the outage performance of the mic tier is inherently much better than that of the mac tier. Consequently, we only have to consider the outage probability of the mac tier as the outage constraint.

The optimal solution $\lambda_{\text {mic }}^{\star}$ to the unconstrained optimization problem $\max _{\lambda_{\min }} \eta_{\mathrm{ESE}}$ is given in Corollary 2. In order to solve the outage-constrained optimization problem (34), we examine the macro-tier outage constraint

$$
Q_{\text {mac }}\left(\lambda_{\text {mic }} ; \lambda_{\text {mac }}, \lambda_{\mathrm{u}}\right)=1+\frac{1}{\rho_{\text {mac }} \exp \left(\rho_{\text {mac }}\right)}-\frac{1}{\rho_{\text {mac }}},
$$

where

$$
\begin{aligned}
& \rho_{\mathrm{mac}}=T_{\mathrm{mac}}^{2 / \alpha} \int_{T_{\mathrm{mac}}^{-2 / \alpha}}^{+\infty} \frac{1}{1+u^{\alpha / 2}} d u, \\
& T_{\text {mac }}=2^{\frac{R_{\text {mac }} \lambda_{u}}{B\left(\lambda_{\text {mac }}+\mu_{\text {mac } \leftarrow \text { mic }}^{-2 / \alpha} \lambda_{\text {mic }}\right)}}-1 .
\end{aligned}
$$


The derivative of $Q_{\text {mac }}$ with respect to $\rho_{\text {mac }}$ satisfies

$$
\begin{aligned}
\frac{\partial Q_{\mathrm{mac}}}{\partial \rho_{\mathrm{mac}}} & =\frac{1}{\rho_{\mathrm{mac}}^{2}}\left(1-\exp \left(-\rho_{\mathrm{mac}}\right)\right)-\frac{1}{\rho_{\mathrm{mac}}} \exp \left(-\rho_{\mathrm{mac}}\right) \\
& >\left.\frac{\partial Q_{\mathrm{mac}}}{\partial \rho_{\mathrm{mac}}}\right|_{\exp }\left(\rho_{\mathrm{mac}}\right)=\rho_{\mathrm{mac}}+1
\end{aligned}
$$

Since $\exp \left(\rho_{\text {mac }}\right)>\rho_{\text {mac }}+1$ always holds for $\rho_{\text {mac }}>0$. This indicates that $Q_{\text {mac }}$ is a monotonically increasing function of $\rho_{\text {mac }}$. From (36) and (37), it is easy to see that $\rho_{\text {mac }}$ increases as $\lambda_{\text {mic }}$ decreases, since $\rho_{\text {mac }}$ increases with $T_{\text {mac }}$ and $T_{\text {mac }}$ decreases as $\lambda_{\text {mic }}$ increases. Therefore, we have

$$
\frac{\partial Q_{\mathrm{mac}}}{\partial \lambda_{\mathrm{mic}}}=\frac{\partial Q_{\mathrm{mac}}}{\partial \rho_{\mathrm{mac}}} \frac{\partial \rho_{\mathrm{mac}}}{\partial \lambda_{\mathrm{mic}}}<0,
$$

which implies that the outage-constrained feasible region of $\lambda_{\text {mic }}$ that satisfies $Q_{\text {mac }}\left(\lambda_{\text {mic }} ; \lambda_{\text {mac }}, \lambda_{\mathrm{u}}\right) \leq \varepsilon_{\text {out }}$ is given by

$$
\lambda_{\text {mic }} \in\left[Q_{\text {mac }}^{-1}\left(\varepsilon_{\text {out }} ; \lambda_{\text {mac }}, \lambda_{\mathrm{u}}\right),+\infty\right),
$$

where $Q_{\operatorname{mac}}^{-1}\left(\cdot ; \lambda_{\text {mac }}, \lambda_{\mathrm{u}}\right)$ denotes the inverse function of $Q_{\text {mac }}\left(\lambda_{\text {mic }} ; \lambda_{\text {mac }}, \lambda_{\mathrm{u}}\right)$.

Moreover, it can be seen from Appendix $\mathrm{C}$ that for $\lambda_{\text {mic }} \in$ $\left[\lambda_{\text {mic }}^{(1)}, \lambda_{\text {mic }}^{\star}\right), \eta_{\mathrm{ESE}}$ is a monotonically increasing function of $\lambda_{\text {mic }}$, while for $\lambda_{\text {mic }} \in\left[\lambda_{\text {mic }}^{\star},+\infty\right), \eta_{\mathrm{ESE}}$ is a monotonically decreasing function of $\lambda_{\text {mic }}$. Based on these properties of $\eta_{\mathrm{ESE}}$ and the outage-constrained feasible region of (40) as well as by defining $\varepsilon_{\text {out }}^{\star}$ as the solution of

$$
Q_{\text {mac }}^{-1}\left(\varepsilon_{\text {out }}^{\star} ; \lambda_{\text {mac }}, \lambda_{\mathrm{u}}\right)=\lambda_{\text {mic }}^{\star},
$$

we readily obtain the outage-constrained optimal solution $\lambda_{\text {mic }}^{\text {out }}$ to the outage-constrained optimization problem (34), as formulated in the following theorem.

Theorem 1. For $\varepsilon_{\text {out }}<\varepsilon_{\text {out }}^{\star}$, i.e., for $Q_{\text {mac }}^{-1}\left(\varepsilon_{\text {out }} ; \lambda_{\text {mac }}, \lambda_{\mathrm{u}}\right)>$ $\lambda_{\text {mic }}^{\star}$, the outage-constrained optimal micro-tier BS density is given by $\lambda_{\text {mic }}^{\text {out } \star}=Q_{\text {mac }}^{-1}\left(\varepsilon_{\text {out }} ; \lambda_{\text {mac }}, \lambda_{\mathrm{u}}\right)$, while for $\varepsilon_{\text {out }}>\varepsilon_{\text {out }}^{\star}$, i.e., for $Q_{\text {mac }}^{-1}\left(\varepsilon_{\text {out }} ; \lambda_{\text {mac }}, \lambda_{\mathrm{u}}\right)<\lambda_{\text {mic }}^{\star}$, the outage-constrained optimal micro-tier BS density is given by $\lambda_{\text {mic }}^{\text {out }}=\lambda_{\text {mic }}^{\star}$.

\section{Numerical and Simulation Results}

Both numerical and simulation results are provided to characterize the ESE of large-scale two-tier HCNs and to verify the accuracy of our ESE performance metrics. Moreover, the APC required by our optimal-EE LUB-aware network design strategy is compared to that of the practical baseline cellular design [39]-[41], for the scenario of an approximate macro BS density of $2 \sim 10 \mathrm{BS} / \mathrm{km}^{2}$. Specifically, we use the energy saving gain to quantify the gain of our optimized design over the baseline design of [39]-[41], which is defined as

$$
\Delta \mathrm{APC}=\frac{\mathrm{APC}_{\text {base }}-\mathrm{APC}_{\mathrm{opt}}}{\mathrm{APC}_{\text {base }}} \text {, }
$$

where $\mathrm{APC}_{\text {base }}$ and $\mathrm{APC}_{\mathrm{opt}}$ denote the $\mathrm{APC}$ required by the baseline design and our optimal design, respectively. Unless otherwise stated, we choose the network parameters as follows: $P_{\mathrm{OM}}^{\mathrm{mac}}=20 \mathrm{~W}, P_{\mathrm{OM}}^{\mathrm{mic}}=0.5 \mathrm{~W}, P_{\max }^{\operatorname{mac}}=40 \mathrm{~W}$, $P_{\max }^{\operatorname{mic}}=2 \mathrm{~W}, \alpha=4, \beta=1.2$ and $\varepsilon_{\text {out }}=0.3$. Note that according to [42], [43], the power consumption of the power amplifier - including the feeder - accounts for approximately $50-80 \%$ of the total power consumption of a BS. Based on this result, we opted for $P_{\mathrm{OM}}^{\operatorname{mac}}=20 \mathrm{~W}$ and $P_{\max }^{\operatorname{mac}}=40 \mathrm{~W}$.

\section{A. Characteristics of Key Performance Metrics}

In Fig. 3, the network's ESE quantified in terms of $\mathrm{bit} / \mathrm{Hz} / \mathrm{Joule}$, as the function of $\lambda_{\text {mac }}$ and $\lambda_{\text {mic }}$ is illustrated. It can be observed from Fig. 3 that for a given $\lambda_{\text {mac }}$, there always exists a unique optimal $\lambda_{\text {mic }}^{\star}$ to maximize $\eta_{\mathrm{ESE}}$, while for a given $\lambda_{\text {mic }}$, there always exists a unique optimal $\lambda_{\text {mac }}^{\star}$ which maximizes $\eta_{\mathrm{ESE}}$. This confirms Corollary 2. It is also worth noting that when the BS density $\lambda_{m}$ in tier $m$ increases or decreases, the optimal density $\lambda_{\widetilde{m}}^{\star}$ in tier $\widetilde{m}$ varies in an inverse direction, where $m=$ mac or mic, while $\widetilde{m}=$ mic or mac. This agrees with Corollary 3.

Fig. 4 illustrates the impact of the required service rates in the two tiers on the network's ESE under varying $\lambda_{\text {mic }}$ as well as given $\lambda_{\text {mac }}=5 \times 10^{-6} \mathrm{BS} / \mathrm{m}^{2}, \lambda_{\mathrm{u}}=4 \times 10^{-4} \mathrm{user} / \mathrm{m}^{2}$, and $\mu=10$. It is clear that increasing $R_{\text {mac }}$ causes a degradation of the network's ESE. This makes sense, since the higher required service rate of macrocell users imposes a higher power consumption due to having a large user-to-macro-BS distance. By contrast, increasing $R_{\text {mic }}$ improves the network's ESE. In other words, increasing the service rate of small cell users consumes less power. Furthermore, the analytical values and simulation results of the network's ESE agree with each other well. This verifies the accuracy of our ESE modeling.

Under the optimal small BS density $\lambda_{\text {mic }}^{\star}$ as well as given $R_{\text {mac }}=R_{\text {mic }}=0.1 \mathrm{Mbit} / \mathrm{s}$ and $\lambda_{\text {mac }}=5 \times 10^{-6} \mathrm{BS} / \mathrm{m}^{2}$, the relationships between the network ESE and the load migration factor as well as the mobile-traffic intensity are shown in Fig. 5 for various system bandwidths. Obviously, increasing $\mu$ decreases the network's ESE, since more users are associated with macro BSs, which consume more transmit power, while a denser $\lambda_{\mathrm{u}}$ improves the ESE to some extent. Furthermore, the network's ESE decreases as $B$ increases. This is because although the network's APC is reduced for an increased $B$, the network's ASE is reduced at a faster rate.

The average aggregate DL transmit power of a macro BS $E\left[P_{\text {mac }}\right]$ versus the mobile traffic intensity $\lambda_{\mathrm{u}}, \lambda_{\text {mic }}$ and $R_{\text {mac }}$ is illustrated in Fig. 6, given $\mu=8$ and $\lambda_{\text {mac }}=$ $5 \times 10^{-6} \mathrm{BS} / \mathrm{m}^{2}$. As expected, $E\left[P_{\text {mac }}\right]$ increases as either $\lambda_{\mathrm{u}}$ or $R_{\mathrm{mac}}$ increases. Furthermore, increasing $\lambda_{\text {mic }}$ causes a larger $E\left[P_{\mathrm{mac}}\right]$ as a result of the increased interference.

Next the average aggregate DL transmit power of small BS $E\left[P_{\text {mic }}\right]$ versus $\lambda_{\text {mic }}, \lambda_{\mathrm{u}}$ and $R_{\text {mic }}$ is shown in Fig. 7, given $\mu=8$ and $\lambda_{\text {mac }}=5 \times 10^{-6} \mathrm{BS} / \mathrm{m}^{2}$. Note that $E\left[P_{\text {mic }}\right]$ decreases upon increasing $\lambda_{\text {mic }}$, which is a result of the reduced small cell size. Furthermore, as expected, increasing $R_{\text {mic }}$ also increases $E\left[P_{\text {mic }}\right]$. Similarly, the average aggregate DL transmit power of macro BS $E\left[P_{\text {mac }}\right]$ versus $\lambda_{\text {mac }}, \lambda_{\mathrm{u}}$ and $R_{\text {mac }}$ is depicted in Fig. 8 , given $\mu=8$ and $\lambda_{\text {mic }}=1 \times 10^{-4} \mathrm{BS} / \mathrm{m}^{2}$. Observe that $R_{\text {mac }}$ has a very significant influence on $E\left[P_{\text {mac }}\right]$.

\section{B. Energy Saving of Optimal Small BS Deployment Strategy}

We now evaluate the energy saving gain of the proposed optimal LUB-aware small-BS deployment strategy over the non-optimal baseline design of [39]-[41]. First, the APC performance of the optimal LUB-aware small-BS deployment design is compared to that of the baseline design in Fig. 9. 


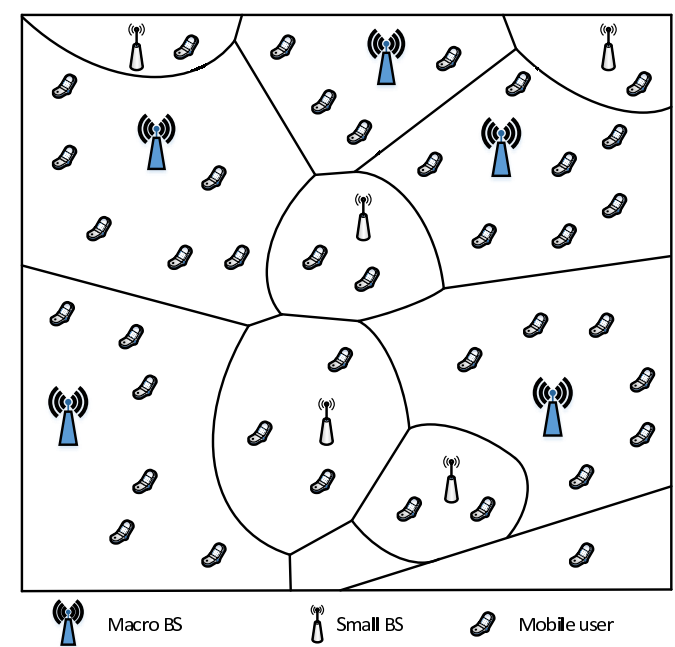

Fig 1: Voronoi topology of a large-scale two-tier heterogeneous cellular network where macro and small BSs as well as mobile users are located

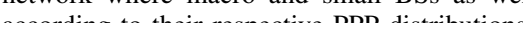

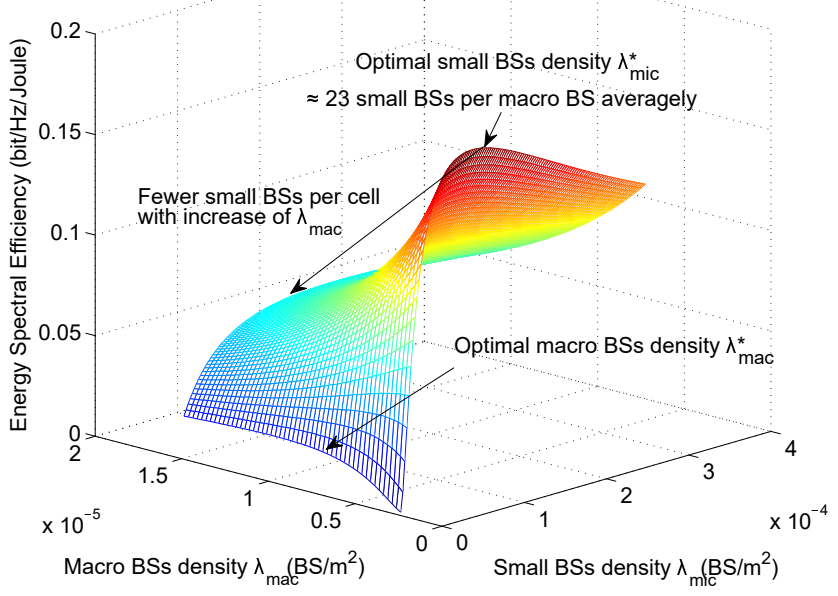

Fig 3: The network ESE $\eta_{\mathrm{ESE}}$ as the function of $\lambda_{\mathrm{mac}}$ and $\lambda_{\mathrm{mic}}$, given $\lambda_{\mathrm{u}}=4 \times 10^{-4}$ user $/ \mathrm{m}^{2}, R_{\mathrm{mac}}=0.3 \mathrm{Mbit} / \mathrm{s}, R_{\mathrm{mic}}=1 \mathrm{Mbit} / \mathrm{s}$ and $\mu=8$.

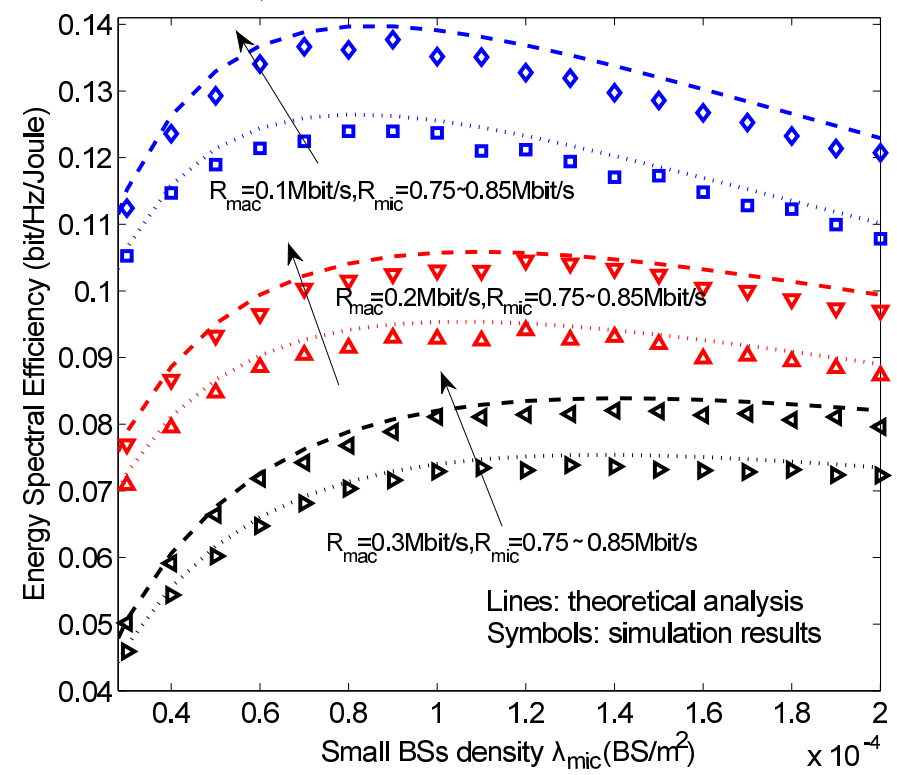

Fig 4: The impacts of $R_{\mathrm{mac}}$ and $R_{\mathrm{mic}}$ on the network ESE $\eta_{\mathrm{ESE}}$ for various $\lambda_{\text {mic }}$, as well as given $\lambda_{\text {mac }}=5 \times 10^{-6} \mathrm{BS} / \mathrm{m}^{2}, \lambda_{\mathrm{u}}=4 \times 10^{-4} \mathrm{user} / \mathrm{m}^{2}$, and $\mu=10$. The arrow indicates increasing $R_{\text {mic }}$.

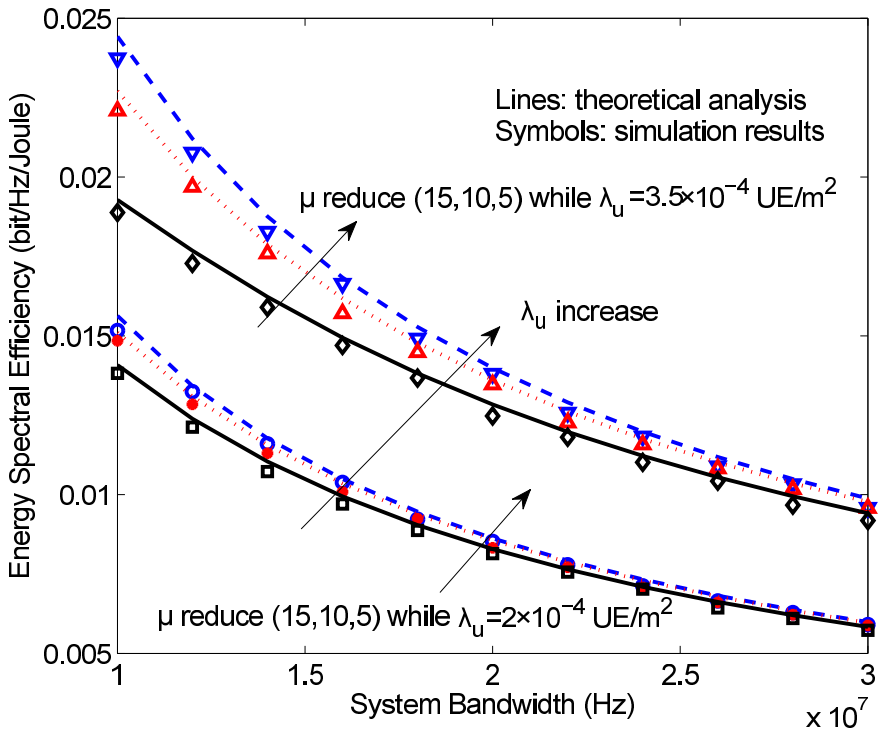

Fig 5: The impacts of $\lambda_{\mathrm{u}}$ and $\mu$ on the network ESE $\eta_{\mathrm{ESE}}$ for various $B$, under the condition of $\lambda_{\text {mic }}^{\star}$ as well as given $R_{\text {mac }}=R_{\text {mic }}=0.1 \mathrm{Mbit} / \mathrm{s}$ and $\lambda_{\text {mac }}=5 \times 10^{-6} \mathrm{BS} / \mathrm{m}^{2}$.

Observe that the proposed optimal LUB-aware small-BS deployment design significantly outperforms the non-optimal baseline design in terms of the network's APC. Moreover, when the mobile traffic intensity $\lambda_{\mathrm{u}}$ is fixed, for the sparser macro BS scenario an increased energy saving gain can be obtained by our optimal LUB-aware small-BS deployment strategy over the baseline design, as clearly seen from Fig. 9. Note that given the fixed $\lambda_{\mathrm{u}}$, for our optimal design, a reduction in $\lambda_{\text {mac }}$ results in an increase in $\lambda_{\text {mic }}^{\star}$. See Corollary 3 . Also when $\lambda_{\text {mac }}$ is fixed, our optimal deployment strategy attains a higher energy saving gain over the baseline design upon increasing $\lambda_{\mathrm{u}}$.

As illustrated in Fig. 10, increasing the coverage range of small BSs by decreasing the LMF $\mu$ can provide increased energy saving gains. This is because decreasing $\mu$ is capable of increasing the efficiency of small BSs deployment by associating more users with the micro cell tier. In Fig. 11, we observe the impact of the service data rate on the energy saving gain, when $R_{\text {mac }}=R_{\text {mic }}$. Clearly, the higher the required service rate, the larger the energy saving gain becomes. This implies that a higher service rate requirement will necessitate denser small BS deployment.

Lastly, in Fig. 12 we investigate how the path-loss exponent $\alpha$ impacts on the achievable energy saving gain. It is widely recognized that a smaller $\alpha$ on one hand is capable of decreasing the transmit power required for users, while on the other hand it can also result in a more serious interference. Observe from Fig. 12 that a smaller $\alpha$ results in a higher energy saving gain for our optimal LUB-aware small-BS deployment strategy over the baseline design.

\section{CONCLUSIONS}

We have proposed an analytical ESE framework for largescale densely deployed multi-tier HCNs. Specifically, we have integrated the key parameters of HCNs, including the per-tier BS densities, the LMFs and the mobile-traffic intensity, into the network's ESE modeling. Significantly, a closed-form ESE expression has been derived, which enables us to analyze the 


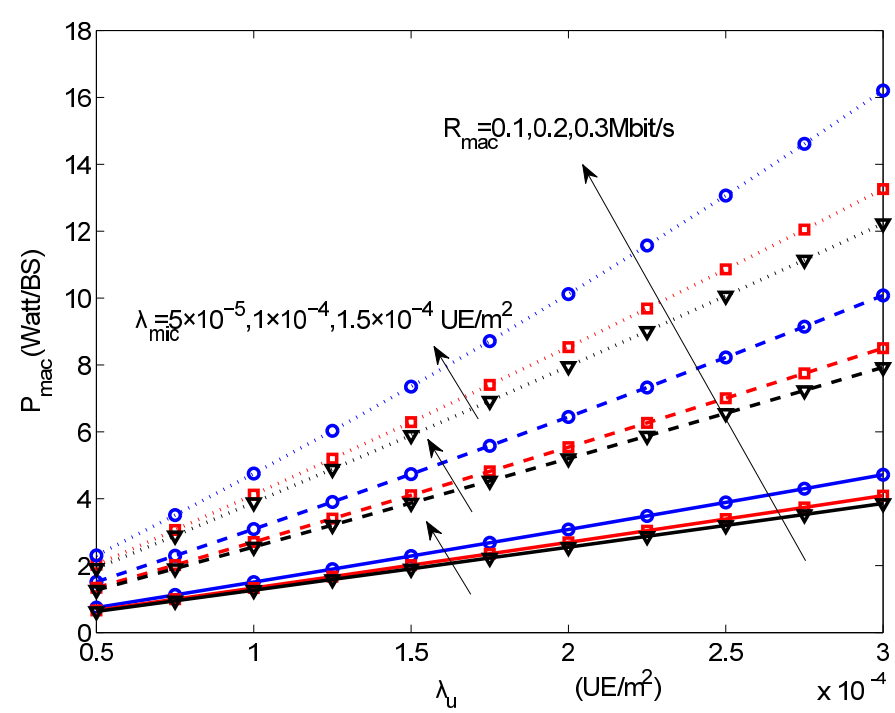

Fig 6: The impacts of $\lambda_{\text {mic }}$ and $R_{\mathrm{mac}}$ on $E\left[P_{\mathrm{mac}}\right]$ for various $\lambda_{\mathrm{u}}$, given $\mu=8$, and $\lambda_{\text {mac }}=5 \times 10^{-6} \mathrm{BS} / \mathrm{m}^{2}$.

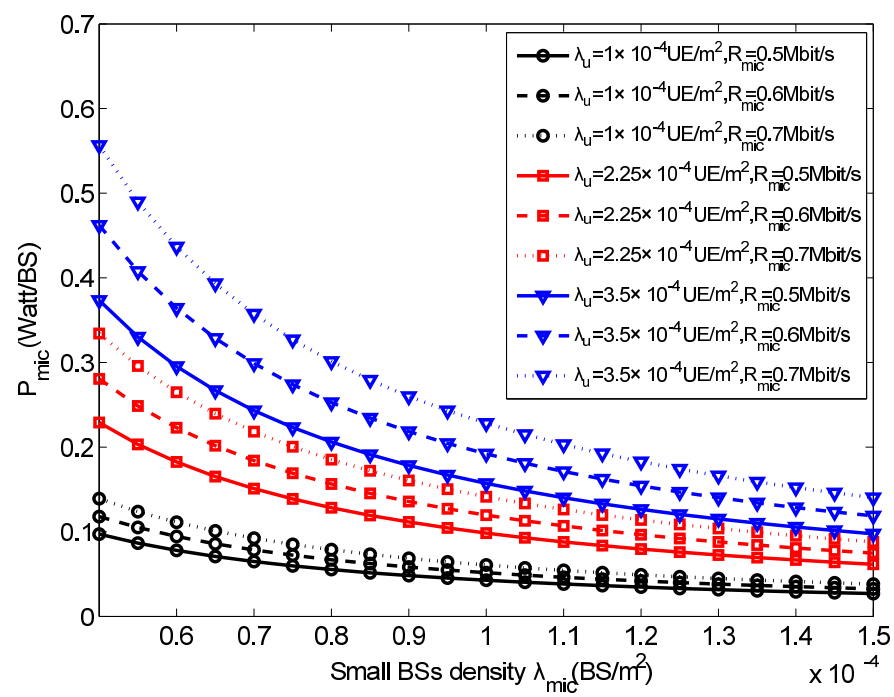

Fig 7: The impacts of $\lambda_{\mathrm{u}}$ and $R_{\text {mic }}$ on $E\left[P_{\text {mic }}\right]$ for various $\lambda_{\text {mic }}$, given $\mu=8$ and $\lambda_{\operatorname{mac}}=5 \times 10^{-6} \mathrm{BS} / \mathrm{m}^{2}$.



Fig 8: The impacts of $\lambda_{\mathrm{u}}$ and $R_{\mathrm{mac}}$ on $E$ [P $\left.P_{\mathrm{mac}}\right]$ for various $\lambda_{\mathrm{mac}}$, given $\mu=8$ and $\lambda_{\text {mic }}=1 \times 10^{-4} \mathrm{BS} / \mathrm{m}^{2}$.

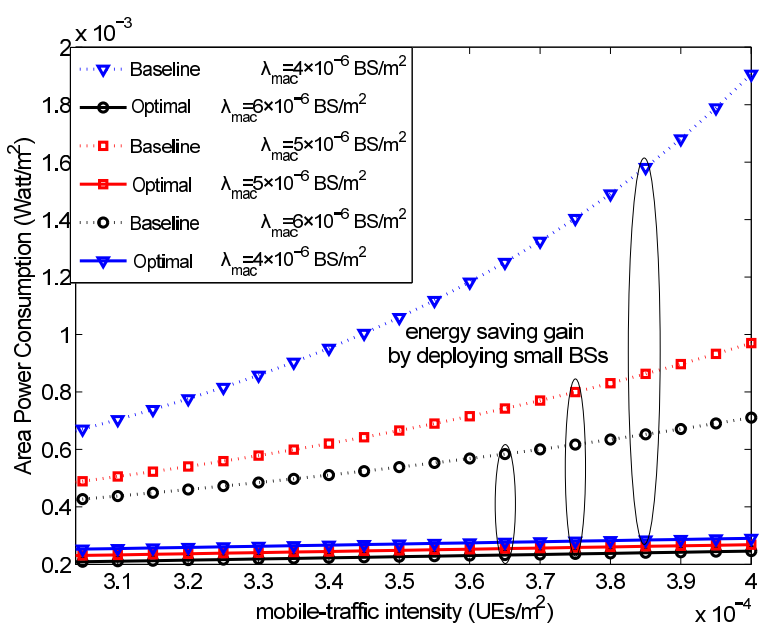

Fig 9: The network APCs as the functions of $\lambda_{\mathrm{u}}$ and $\lambda_{\mathrm{mac}}$ for the optimal and non-optimal small-BS deployment designs, given $R_{\mathrm{mac}}=R_{\mathrm{mic}}=$ $0.3 \mathrm{Mbit} / \mathrm{s}$.

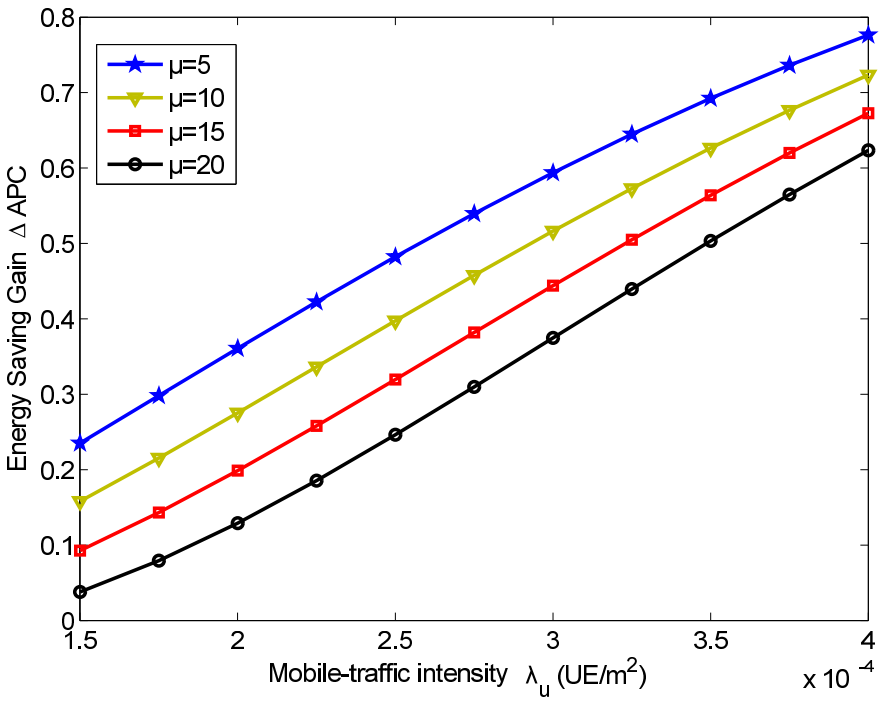

Fig 10: Energy saving gain attained by the optimal LUBs-aware small-BS deployment strategy over the baseline design as the function of $\lambda_{\mathrm{u}}$ for different $\mu$, given $B=10 \mathrm{Mbit} / \mathrm{s}, \lambda_{\mathrm{mac}}=5 \times 10^{-6} \mathrm{BS} / \mathrm{m}^{2}$, and $R_{\mathrm{mac}}=R_{\mathrm{mic}}=$ $0.3 \mathrm{Mbit} / \mathrm{s}$.

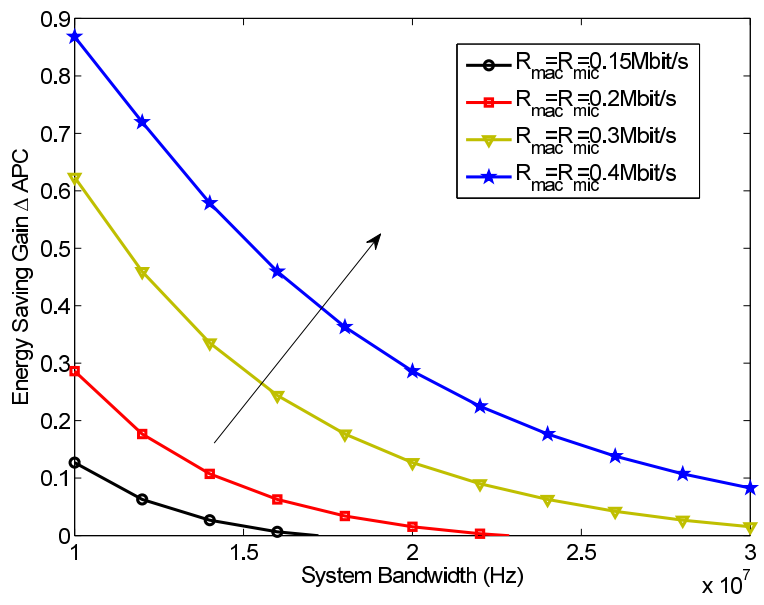

Fig 11: Energy saving gain attained by the optimal LUBs-aware small-BS deployment strategy over the baseline design as the function of $B$ for various $R_{\mathrm{mac}}=R_{\text {mic }}$ values, given $\lambda_{\mathrm{mac}}=5 \times 10^{-6} \mathrm{BS} / \mathrm{m}^{2}, \mu=20$ and $\lambda_{\mathrm{u}}=4 \times 10^{-4} \mathrm{UE} / \mathrm{m}^{2}$. 
properties of multi-tier HCNs. Moreover, an optimal LUBaware ESE BS deployment strategy was conceived for twotier macro-small HCNs, which maximizes the network's ESE under outage constraints. The accuracy of our ESE modeling has been verified by our simulations results. This study has qualitatively and quantitatively shown the significant energy saving gains achievable by our proposed optimal LUBs-aware BS deployment design conceived for large-scale HCNs, and our results have provided valuable design insights for the ultradense $\mathrm{HCN}$ of the near future.

\section{APPENDIX}

\section{A. Proof of Proposition 1}

Proof. Given typical UE $u_{j, m}$ in position $z$ and associated to $\mathrm{BS} b_{m}$ in tier $m$, which requests rate $R_{j, m}$ and is allocated with subband $B_{j, m}$, its required DL transmit power conditioning on $r_{m}=\left\|z-b_{m}\right\|$ and interference topology $\left(\bigcup_{\widetilde{m}} \Psi_{\widetilde{m}}\right) \backslash b_{m}$ is given by

$$
\begin{aligned}
& E\left[P_{j, m} \mid r_{m},\left(\bigcup_{\widetilde{m}} \Psi_{\widetilde{m}}\right) \backslash b_{m}\right]=\frac{\left(2^{R_{j, m} / B_{j, m}}-1\right)}{r_{m}^{-\alpha}} E\left[I_{j, m}\right] \\
& =\frac{\left(2^{R_{j, m} / B_{j, m}}-1\right)}{r_{m}^{-\alpha}} E\left[\sum_{\widetilde{m}=1}^{M} \sum_{b_{k} \in \Psi_{\widetilde{m}, b_{k} \neq b_{m}}} \frac{g_{j}^{k} P_{\max }^{\widetilde{m}}}{N_{m} r_{k}^{\alpha}}\right] .
\end{aligned}
$$

In deriving (43) from (2) and (3), we have utilized $E\left[g_{j}^{m}\right]=1$. The moment generating function (MGF) of $I_{j, m}$ is [34]

$$
\begin{aligned}
\mathcal{M}_{I}(s) & =E\left[\exp \left(-\sum_{\widetilde{m}=1}^{M} \sum_{b_{k} \in \Psi_{\widetilde{m}}, b_{k} \neq b_{m}} s \frac{g_{j}^{k} P_{\max }^{\widetilde{m}}}{N_{m} r_{k}^{\alpha}}\right)\right] \\
& =E\left[\prod_{\widetilde{m}=1}^{M} \prod_{b_{k} \in \Psi_{\widetilde{m}}, b_{k} \neq b_{m}} \exp \left(-s \frac{g_{j}^{k} P_{\max }^{\tilde{m}}}{N_{m} r_{k}^{\alpha}}\right)\right] .
\end{aligned}
$$

Now consider the aggregate interference power $I^{\prime}$ from the BSs in all the tiers where each tier has a finite region with the inner radius $r_{\widetilde{m}}$ and the outer radius $r_{\mathrm{bn}}^{\widetilde{m}}=r_{\mathrm{bn}}$ for tier $\widetilde{m}$. We



Fig 12: Energy saving gain attained by the optimal LUBs-aware small-BS deployment strategy over the baseline design as the function of $B$ for various $\alpha$ values, given $\lambda_{\text {mac }}=5 \times 10^{-6} \mathrm{BS} / \mathrm{m}^{2}, \mu=20, \lambda_{\mathrm{u}}=4 \times 10^{-4} \mathrm{UE} / \mathrm{m}^{2}$, and $R_{\mathrm{mac}}=R_{\mathrm{mic}}=0.3 \mathrm{Mbit} / \mathrm{s}$. have $I^{\prime}=\sum_{\widetilde{m}=1}^{M} I_{\widetilde{m}}^{\prime}$, where $I_{\widetilde{m}}^{\prime}$ is the interference from tier $\widetilde{m}$. The MGF of $I_{\widetilde{m}}^{\prime}$ is given by [14], [35]

$$
\mathcal{M}_{I_{\bar{m}}^{\prime}}(s)=\exp \left(2 \pi \lambda_{\widetilde{m}} \int_{r_{\widetilde{m}}}^{r_{\mathrm{bn}}}\left(1-\exp \left(-\frac{s P_{\max }^{\widetilde{m}}}{N_{m} t^{\alpha}}\right)\right) t d t\right) \text {. }
$$

Again $E\left[g_{j}^{k}\right]=1$ is utilized in deriving (45). Hence, the MGF of $I^{\prime}$ is given by

$$
\mathcal{M}_{I^{\prime}}(s)=\prod_{\widetilde{m}} \mathcal{M}_{I_{\widehat{m}}^{\prime}}(s) .
$$

According to the well-known property of MGF, we have

$$
\begin{aligned}
E\left[I^{\prime}\right] & =-\left.\frac{\partial}{\partial s} \ln \mathcal{M}_{I^{\prime}}(s)\right|_{s=0} \\
& =\sum_{\widetilde{m}} \frac{2 \pi \lambda_{\widetilde{m}} P_{\max }^{\widetilde{m}}}{N_{m}} \frac{r_{\mathrm{bn}}^{2-\alpha}-r_{\widetilde{m}}^{2-\alpha}}{(2-\alpha)},
\end{aligned}
$$

assuming $\alpha>2$. Clearly, $E\left[I_{j, m}\right]=\lim _{r_{\mathrm{bn}} \rightarrow \infty} E\left[I^{\prime}\right]$. By letting $r_{\mathrm{bn}} \rightarrow \infty$ in (47) and substituting the result into (43) as well as utilizing $\mu_{m \leftarrow \widetilde{m}}=\left(\frac{r_{\widetilde{m}}}{r_{m}}\right)^{-\alpha}$ [26], [27], we obtain

$$
\begin{aligned}
E\left[P_{j, m} \mid r_{m}\right]= & \frac{2 \pi \lambda_{m} P_{\max }^{m} r_{m}^{2}\left(2^{R_{j, m} / B_{j, m}}-1\right)}{N_{m}(\alpha-2)} \\
& \times\left(\sum_{\widetilde{m}=1}^{M} \frac{\lambda_{\widetilde{m}} P_{\max }^{\widetilde{m}}}{\lambda_{m} P_{\max }^{m}} \mu_{m \leftarrow \widetilde{m}}^{(\alpha-2) / \alpha}\right) .
\end{aligned}
$$

The user distribution within $V_{m}$ can be accurately approximated by a uniform distribution ${ }^{2}$. Further approximate cell $V_{m}$ with area $A_{m}$ by the circle having area $A_{m}=\pi R_{\text {ave }}^{2}$, where $R_{\text {ave }}$ is the radius of the cell. Since $N_{m}=A_{m} \lambda_{\mathrm{u}}$, $R_{\mathrm{ave}}=\sqrt{\frac{N_{m}}{\pi \lambda_{\mathrm{u}}}}$. Thus the probability density function (PDF) of the distance $x$ between user $u_{j, m}$ and $\mathrm{BS} b_{m}$ is $f(x)=\frac{2 x}{R_{\text {ave }}^{2}}$, and therefore we have

$$
\begin{aligned}
& E\left[P_{j, m}\right]=\int_{0}^{R_{\text {ave }}} \frac{2 r_{m} E\left[P_{j, m} \mid r_{m}\right]}{R_{\text {ave }}^{2}} d r_{m} \\
& =\frac{\lambda_{m}\left(2^{R_{j, m} / B_{j, m}}-1\right)}{(\alpha-2) \lambda_{\mathrm{u}}} \sum_{\widetilde{m}=1}^{M} \frac{\lambda_{\widetilde{m}} P_{\max }^{\widetilde{m}} \mu_{m \leftarrow \widetilde{m}}^{(\alpha-2) / \alpha}}{\lambda_{m}} .
\end{aligned}
$$

This completes the proof.

\section{B. Proof of Proposition 2}

Proof. Given the typical cell $V_{m}$ centered by $b_{m}$, the average aggregate DL transmit power conditioned on the cell area of $\left|V_{m}\right|=A_{m}$ can be expressed as

$$
\begin{aligned}
E\left[P_{m} \mid A_{m}\right] & =\sum_{u_{i} \in \Psi^{\mathrm{u}}} \mathcal{P}_{u_{i}} \mathcal{I}\left\{u_{i} \in \varphi_{m}\right\} \\
& =\sum_{N_{m}=1}^{\infty}\left(\sum_{j=1}^{N_{m}} E\left[P_{j, m}\right]\right) g\left(N_{m}\right),
\end{aligned}
$$

where $\mathcal{P}_{u_{i}}$ denotes the DL transmit power for user $u_{i}$ and $\mathcal{I}\{\}$ is the indicator function, which is equal to 1 when the

\footnotetext{
${ }^{2}$ Although the user distribution in the whole network is modelled by a nonuniform PPP process, the distribution of users in a single cell can accurately be modelled by a uniform distribution.
} 
condition inside the bracket is satisfied and 0 otherwise, while $g\left(N_{m}\right)$ denotes the PMF of the number of users in $V_{m}$, which is given by $g\left(N_{m}\right)=\left(\lambda_{\mathrm{u}} A_{m}\right)^{N_{m}} \exp \left(-\lambda_{\mathrm{u}} A_{m}\right) / N_{m}$ ! for $N_{m} \geq 1$ [36].

Since all the UEs in $V_{m}$ have the identical rate of $R_{j, m}=$ $R_{m}$, from (5), we have

$$
E\left[P_{j, m}\right]=\frac{P_{\max }^{m}\left(2^{N_{m} R_{m} / B}-1\right)}{(\alpha-2) \lambda_{\mathrm{u}}} \sum_{\widetilde{m}=1}^{M} \lambda_{\widetilde{m}} \mu_{m \leftarrow \widetilde{m}}^{-2 / \alpha},
$$

where $\mu_{m \leftarrow \widetilde{m}}=P_{\max }^{m} / P_{\max }^{\widetilde{m}}$ [26], [27] is utilized. Further note that the average value of $N_{m}$ is

$$
E\left[N_{m}\right]=\frac{\lambda_{\mathrm{u}}}{\sum_{\widetilde{m}=1}^{M} \lambda_{\widetilde{m}} \mu_{m \leftarrow \widetilde{m}}^{-2 / \alpha}} .
$$

Note that $1 / \sum_{\widetilde{m}=1}^{M} \lambda_{\widetilde{m}} \mu_{m \leftarrow \widetilde{m}}^{-2 / \alpha}$ in (52) is approximately the average coverage area of $b_{m}$ for the $M$-tier HCN [26], [27]. Thus the average aggregate DL transmit power conditioned on $A_{m}$ can be expressed as

$$
\begin{aligned}
& E\left[P_{m} \mid A_{m}\right]=E\left[N_{m} E\left[P_{j, m}\right]\right] \\
& =\sum_{N_{m}=1}^{\infty} \frac{P_{\max }^{m}\left(2^{N_{m} R_{m} / B}-1\right)}{(\alpha-2)} \frac{\left(\lambda_{\mathrm{u}} A_{m}\right)^{N_{m}}}{N_{m} ! \exp \left(\lambda_{\mathrm{u}} A_{m}\right)} .
\end{aligned}
$$

Consequently,

$$
E\left[P_{m}\right]=\int_{A_{m}>0} E\left[P_{m} \mid A_{m}\right] \mathcal{G}\left(A_{m}\right) d A_{m},
$$

where $\mathcal{G}\left(A_{m}\right)$ is the PDF of $A_{m}$. According to [37],

$$
\mathcal{G}\left(A_{m}\right)=\frac{K^{K}\left(\lambda_{m}+\sum_{\tilde{m} \neq m} \lambda_{\widetilde{m}} \mu_{m \leftarrow \widetilde{m}}^{-2 / \alpha}\right)^{K} A_{m}^{K-1}}{\Gamma(k) \exp \left(K\left(\lambda_{m}+\sum_{\tilde{m} \neq m} \lambda_{\widetilde{m}} \mu_{m \leftarrow \widetilde{m}}^{-2 / \alpha}\right) A_{m}\right)},
$$

where $K=3.57$ [38], and the Gamma function $\Gamma(x)=$ $\int_{0}^{+\infty} t^{x-1} \exp (-t) d t$. Substituting (53) and (55) into (54) and completing the resulting integration yields (6). The detailed final part of this proof is similar to Appendix C of [14].

\section{Proof of Proposition 4}

Proof. By taking the derivative of the network-level ESE with respect to $\lambda_{m}$, we obtain

$$
\frac{\partial \eta_{\mathrm{ESE}}}{\partial \lambda_{m}}=-\frac{N_{\eta}}{\left(D_{\eta}\right)^{2}} \Omega,
$$

where $N_{\eta}$ and $D_{\eta}$ are the numerator and denominator of $\eta_{\mathrm{ESE}}$, respectively,

$$
\Omega=\frac{1-(1+K) \Upsilon_{m}}{\left(1-\Upsilon_{m}\right)^{K+1}}+\sum_{\widetilde{m} \neq m} \mu_{m \leftarrow \widetilde{m}}^{\frac{2-\alpha}{\alpha}} \frac{1-(1+K) \Upsilon_{\widetilde{m}}}{\left(1-\Upsilon_{\widetilde{m}}\right)^{K+1}}-\gamma_{m}^{(M)},
$$

and $\gamma_{m}^{(M)}$ is given in (20), while $\Upsilon_{m}$ and $\Upsilon_{\widetilde{m}}$ are given in (18) and (19), respectively, but with $\lambda_{m}^{\star}$ replaced by $\lambda_{m}$. Since $N_{\eta}>0$ and $D_{\eta}>0$, the sign of $\frac{\partial \eta_{\mathrm{ESE}}}{\partial \lambda_{m}}$ is equal to the sign of $-\Omega$.
Clearly, from (18), there exists a $\lambda_{m}^{(1)}$ such that $\Upsilon_{m} \rightarrow 1^{-}$ as $\lambda_{m} \rightarrow \lambda_{m}^{(1)+}$. Therefore,

$$
\lim _{\lambda_{m} \rightarrow \lambda_{m}^{(1)+}} \Omega=-\infty \Rightarrow \lim _{\lambda_{m} \rightarrow \lambda_{m}^{(1)+}} \frac{\partial \eta_{\mathrm{ESE}}}{\partial \lambda_{m}}>0 .
$$

In fact, from (18), we have

$$
\lambda_{m}^{(1)}=\left(2^{R_{m} / B}-1\right) \frac{\lambda_{\mathrm{u}}}{K}-\sum_{\widetilde{m} \neq m} \lambda_{\widetilde{m}} \mu_{m \leftarrow \widetilde{m}}^{-2 / \alpha} .
$$

Also $\Upsilon_{m} \rightarrow 0$ and $\Upsilon_{\widetilde{m}} \rightarrow 0$ as $\lambda_{m} \rightarrow+\infty$. Therefore, we have

$$
\begin{aligned}
& \lim _{\lambda_{m} \rightarrow+\infty} \frac{1-(1+K) \Upsilon_{m}}{\left(1-\Upsilon_{m}\right)^{K+1}}=1, \\
& \lim _{\lambda_{m} \rightarrow+\infty} \sum_{\widetilde{m} \neq m} \mu_{m \leftarrow \widetilde{m}}^{\frac{2-\alpha}{\alpha}} \frac{1-(1+K) \Upsilon_{\widetilde{m}}}{\left(1-\Upsilon_{\widetilde{m}}\right)^{K+1}}=\sum_{\widetilde{m} \neq m} \mu_{m \leftarrow \widetilde{m}}^{\frac{2-\alpha}{\alpha}},
\end{aligned}
$$

which imply that

$$
\lim _{\lambda_{m} \rightarrow+\infty} \Omega=\frac{P_{\mathrm{OM}}^{m}}{P_{\max }^{m}}(\alpha-2)>0 \Rightarrow \lim _{\lambda_{m} \rightarrow+\infty} \frac{\partial \eta_{\mathrm{ESE}}}{\partial \lambda_{m}}<0 .
$$

Next, it can be shown that $\frac{\partial \Omega}{\partial \lambda_{m}}>0$. This implies that $\Omega$ is a monotonically increasing function of $\lambda_{m}$. Therefore, there exists an unique $\lambda_{m}^{\star} \in\left[\lambda_{m}^{(1)},+\infty\right)$ which maximizes $\eta_{\mathrm{ESE}}$. Setting (57) to zero completes the proof.

\section{Proof of Corollary 3}

Proof. First, we introduce

$$
\Theta\left(\lambda_{m}^{\star}, \lambda_{\widetilde{m}}\right)=\underbrace{\frac{1-(1+K) \bar{\Upsilon}_{m}}{\left(1-\bar{\Upsilon}_{m}\right)^{K+1}}}_{\delta_{1}}+\underbrace{\mu_{m \leftarrow \widetilde{m}}^{\frac{2-\alpha}{\alpha}} \frac{1-(1+K) \bar{\Upsilon}_{\widetilde{m}}}{\left(1-\bar{\Upsilon}_{\widetilde{m}}\right)^{K+1}}}_{\delta_{2}} .
$$

The derivative of $\Theta$ with respect to $\lambda_{m}^{\star}$ satisfies

$$
\begin{aligned}
& \frac{\partial \Theta\left(\lambda_{m}^{\star}, \lambda_{\widetilde{m}}\right)}{\partial \lambda_{m}^{\star}}=\frac{\partial \delta_{1}}{\partial \bar{\Upsilon}_{m}} \frac{\partial \bar{\Upsilon}_{m}}{\partial \lambda_{m}^{\star}}+\frac{\partial \delta_{2}}{\partial \bar{\Upsilon}_{\widetilde{m}}} \frac{\partial \bar{\Upsilon}_{\tilde{m}}}{\partial \lambda_{m}^{\star}} \\
& =\left(\frac{-K(K+1) \bar{\Upsilon}_{m}}{\left(1-\bar{\Upsilon}_{m}\right)^{K+2}}\right)\left(\frac{-\left(2^{R_{m} / B}-1\right) \lambda_{\mathrm{u}}}{K\left(\lambda_{m}^{\star}+\lambda_{\widetilde{m}} \mu_{m \leftarrow \widetilde{m}}^{-\frac{2}{\alpha}}\right)^{2}}\right)+ \\
& \left(\frac{-K(K+1) \bar{\Upsilon}_{\widetilde{m}} \mu_{m \leftarrow \widetilde{m}}^{\frac{2-\alpha}{\alpha}}}{\left(1-\bar{\Upsilon}_{\tilde{m}}\right)^{K+2}}\right)\left(\frac{-\mu_{\widetilde{m} \leftarrow m}^{-\frac{2}{\alpha}}\left(2^{R_{m} / B}-1\right) \lambda_{\mathrm{u}}}{K\left(\lambda_{\widetilde{m}}+\lambda_{m}^{\star} \mu_{\widetilde{m} \leftarrow m}^{-\frac{2}{\alpha}}\right)^{2}}\right)>0 .
\end{aligned}
$$

Similarly, we have

$$
\begin{aligned}
& \frac{\partial \Theta\left(\lambda_{m}^{\star}, \lambda_{\widetilde{m}}\right)}{\partial \lambda_{\widetilde{m}}}=\frac{\partial \delta_{1}}{\partial \bar{\Upsilon}_{m}} \frac{\partial \bar{\Upsilon}_{m}}{\partial \lambda_{\widetilde{m}}}+\frac{\partial \delta_{2}}{\partial \bar{\Upsilon}_{\widetilde{m}}} \frac{\partial \bar{\Upsilon}_{\widetilde{m}}}{\partial \lambda_{\widetilde{m}}} \\
& =\left(\frac{-K(K+1) \bar{\Upsilon}_{m}}{\left(1-\bar{\Upsilon}_{m}\right)^{K+2}}\right)\left(\frac{-\left(2^{R_{m} / B}-1\right) \lambda_{\mathrm{u}} \mu_{m \leftarrow \widetilde{m}}^{-\frac{2}{\alpha}}}{K\left(\lambda_{m}^{\star}+\lambda_{\widetilde{m}} \mu_{m \leftarrow \widetilde{m}}^{-\frac{2}{\alpha}}\right)^{2}}\right)+ \\
& \left(\frac{-K(K+1) \bar{\Upsilon}_{\widetilde{m}} \mu_{m \leftarrow \widetilde{m}}^{\frac{2-\alpha}{\alpha}}}{\left(1-\bar{\Upsilon}_{\widetilde{m}}\right)^{K+2}}\right)\left(\frac{-\left(2^{R_{m} / B}-1\right) \lambda_{\mathrm{u}}}{K\left(\lambda_{\widetilde{m}}+\lambda_{m}^{\star} \mu_{\widetilde{m} \leftarrow m}^{-\frac{2}{\alpha}}\right)^{2}}\right)>0 .
\end{aligned}
$$


The conditions (64) and (65) indicate that $\Theta\left(\lambda_{m}^{\star}, \lambda_{\widetilde{m}}\right)$ is a monotonically increasing function of $\lambda_{m}^{\star}$ and $\lambda_{\widetilde{m}}$, when the network operates near the optimal ESE status of $\lambda_{m}^{\star}$. Therefore, to maintain the network at a near optimal ESE status, increasing $\lambda_{\widetilde{m}}$ has to be compensated by reducing $\lambda_{m}^{\star}$, and vice versa. This completes the proof.

\section{E. Proof of Proposition 5}

Proof. Firstly, express the network's $\eta_{\mathrm{ESE}}$ (11) as

$$
\eta_{\mathrm{ESE}}=\frac{N_{\eta}}{D_{\eta}}=\left(\sum_{m=1}^{M} \frac{1}{B} \frac{R_{m} \lambda_{m}}{\left(\sum_{\widetilde{m}=1}^{M} \lambda_{\widetilde{m}} \mu_{m \leftarrow \widetilde{m}}^{-2 / \alpha}\right)}\right) \frac{1}{D_{\eta}} .
$$

The derivative of $\eta_{\mathrm{ESE}}$ with respect to $\lambda_{\mathrm{u}}$ is

$$
\frac{\partial \eta_{\mathrm{ESE}}}{\partial \lambda_{\mathrm{u}}}=-\frac{N_{\eta}}{D_{\eta}^{2}} \frac{\partial D_{\eta}}{\partial \lambda_{\mathrm{u}}}
$$

where

$$
\begin{aligned}
\frac{\partial D_{\eta}}{\partial \lambda_{\mathrm{u}}}= & \frac{1}{\left(\lambda_{\mathrm{u}}\right)^{2}} \sum_{m=1}^{M}\left(\frac{K P_{\max }^{m} \beta_{m} \lambda_{m} \lambda_{\mathrm{u}} S_{m}}{(\alpha-2)\left(1-S_{m} \lambda_{\mathrm{u}}\right)^{K+1}}\right. \\
& \left.-\frac{P_{\max }^{m} \beta_{m} \lambda_{m}}{(\alpha-2)\left(1-S_{m} \lambda_{\mathrm{u}}\right)^{K}}+\frac{\lambda_{m} \beta_{m} P_{\max }^{m}}{(\alpha-2)}-\lambda_{m} P_{\mathrm{OM}}^{m}\right) \\
= & \frac{1}{\left(\lambda_{\mathrm{u}}\right)^{2}} D^{\prime} .
\end{aligned}
$$

Clearly, the sign of $\frac{\partial D_{\eta}}{\partial \lambda_{u}}$ is determined by the sign of $D^{\prime}$, and furthermore

$$
\begin{aligned}
& \lim _{\lambda_{\mathrm{u}} \rightarrow 0^{+}} D^{\prime}=-\sum_{m=1}^{M} \lambda_{m} P_{\mathrm{OM}}^{m}<0, \\
& \lim _{\lambda_{\mathrm{u}} \rightarrow{\frac{1}{S_{m}}}^{-}} D^{\prime}=+\infty+\sum_{m=1}^{M}\left(\frac{\lambda_{m} \beta_{m} P_{\max }^{m}}{(\alpha-2)}-\lambda_{m} P_{\mathrm{OM}}^{m}\right)>0 .
\end{aligned}
$$

Since the derivative of $D^{\prime}$ with respect to $\lambda_{\mathrm{u}}$

$$
\frac{\partial D^{\prime}}{\partial \lambda_{\mathrm{u}}}=\sum_{m=1}^{M} \frac{K P_{\max }^{m} \beta_{m} \lambda_{m} \lambda_{\mathrm{u}}(K+1) S_{m}^{2}}{(\alpha-2)\left(1-S_{m} \lambda_{\mathrm{u}}\right)^{K+2}}>0,
$$

$D^{\prime}$ is a monotonically increasing function of $\lambda_{u}$.

Based on (69) to (71), there must exist a unique $\lambda_{\mathrm{u} \star} \in$ $\left[0, \frac{1}{S_{m}}\right)$ to enable $\left.\frac{\partial D_{\eta}}{\partial \lambda_{\mathrm{u}}}\right|_{\lambda_{\mathrm{u} \star}}=0$. That is, there always exists the unique optimal $\lambda_{\mathrm{u} \star} \in\left[0, \frac{1}{S_{m}}\right)$ as the solution of (26), which maximizes $\eta_{\mathrm{ESE}}$.

\section{F. Proof of Proposition 6}

Proof. The outage probability of user $u_{j, m}$ located at $z$, i.e., conditioned on $r_{m}=\left\|z-b_{m}\right\|$, is given by

$$
Q_{j, m}\left[r_{m}\right]=1-\operatorname{Pr}\left(P_{j, m} \leq P_{\max }^{m} / N_{m} \mid r_{m}\right) .
$$

From (2), $P_{j, m}=\left(2^{R_{j, m} / B_{j, m}}-1\right) I_{j, m} r_{m}^{\alpha} / g_{j}^{m}$, and hence according to [23] we have

$$
Q_{j, m}\left[r_{m}\right]=1-\left.\mathcal{L}_{I_{j, m}}(s)\right|_{s=\frac{N_{m}}{P_{\max }^{m}} T_{j, m} r_{m}^{\alpha}},
$$

where $\mathcal{L}_{I_{j, m}}(s)$ is the Laplace transform of $I_{j, m}$, and $T_{j, m}=$ $\left(2^{R_{j, m} / B_{j, m}}-1\right)$. From (3),

$$
I_{j, m}=\sum_{\widetilde{m}} \sum_{b_{k} \in \Psi_{\widetilde{m}}, b_{k} \neq b_{m}} P_{\max }^{\widetilde{m}} g_{j}^{k} r_{k}^{\alpha} / N_{m},
$$

in which $\frac{P_{\max }^{\widetilde{m}}}{N_{m}} g_{j}^{k} \sim \exp \left(N_{m} / P_{\max }^{\widetilde{m}}\right)$. Therefore, according to Appendix B of [23], we have

$$
\mathcal{L}_{I_{j, m}}\left(\frac{N_{m}}{P_{\max }^{m}} T_{j, m} r_{m}^{\alpha}\right)=\prod_{\widetilde{m}} \exp \left(-\pi \mu_{m \leftarrow \widetilde{m}}^{-2 / \alpha} r_{m}^{2} \lambda_{\widetilde{m}} \rho_{j, m}\right),
$$

where $\rho_{j, m}$ is defined in (29). Noting that the PDF of $r$ is $f(r)=\frac{2 r}{R_{\text {ave }}^{2}}, 0 \leq r \leq R_{\text {ave }}$, with $R_{\text {ave }}^{2}=\frac{N_{m}}{\pi \lambda_{\mathrm{u}}}$, we have

$$
Q_{j, m}=1-\int_{0}^{R_{\text {ave }}} \frac{2 x}{R_{\text {ave }}^{2}} \exp \left(-\pi \sum_{\widetilde{m}=1}^{M} \mu_{m \leftarrow \widetilde{m}}^{-2 / \alpha} \lambda_{\widetilde{m}} \rho_{j, m} x^{2}\right) d x .
$$

Completing the integration (76) leads to (28).

\section{REFERENCES}

[1] J. G. Andrews, et al., "What will 5G be?" IEEE J. Sel. Areas Commun., vol. 32, no. 6, pp. 1065-1082, Jun. 2014.

[2] J. G. Andrews, H. Claussen, M. Dohler, S. Rangan, and M. C. Reed, "Femtocells: past, present, and future," IEEE J. Sel. Areas Commun. vol. 30, no. 3, pp. 497-508, Apr. 2012.

[3] A. P. Bianzino, C. Chaudet, D. Rossi, and J.-L. Rougier, "A survey of green networking research," IEEE Commun. Surveys Tuts., vol. 14, no. 1, pp. 3-20, 2012.

[4] C. Han, et al., "Green radio: radio techniques to enable energy-efficient wireless networks," IEEE Commun. Mag., vol. 49, no. 6, pp. 46-54, Jun. 2011.

[5] Z. Niu, Y. Wu, J. Gong, and Z. Yang, "Cell zooming for cost-efficient green cellular networks," IEEE Commun. Mag., vol. 48, no. 11, pp. 74 79, Nov. 2010.

[6] N. Saxena, B. J. R. Sahu, and Y. S. Han, "Traffic-aware energy optimization in green LTE cellular systems," IEEE Commun. Lett., vol. 18, no. 1, pp. 38-41, Jan. 2014.

[7] E. Oh, K. Son, and B. Krishnamachari, "Dynamic base station switchingon/off strategies for green cellular networks," IEEE Trans. Wireless Commun., vol. 12, no. 5, pp. 2126-2136, May 2013.

[8] K. T. K. Cheung, S. Yang, and L. Hanzo, "Achieving maximum energyefficiency in multi-relay OFDMA cellular networks: a fractional programming approach," IEEE Trans. Commun., vol. 61, no. 7, pp. 27462757, Jul. 2013.

[9] K. T. K. Cheung, S. Yang, and L. Hanzo, "Spectral and energy spectral efficiency optimization of joint transmit and receive beamforming based multi-relay MIMO-OFDMA cellular networks," IEEE Trans. Wireless Commun., vol. 13, no. 11, pp. 6147-6165, Nov. 2014.

[10] W. Jing, Z. Lu, X. Wen, Z. Hu, and S. Yang, "Flexible resource allocation for joint optimization of energy and spectral efficiency in OFDMA multicell networks," IEEE Commun. Lett., vol. 19, no. 3, pp. 451-454, Mar. 2015.

[11] K. T. K. Cheung, S. Yang, and L. Hanzo, "Distributed energy spectral efficiency optimization for partial/full interference alignment in multiuser multi-relay multi-cell MIMO systems," IEEE Trans. Signal Process., vol. 64, no. 4, pp. 882-896, Feb. 2016.

[12] T. Abräo, et al., "Energy efficient OFDMA networks maintaining statistical QoS guarantees for delay-sensitive traffic," IEEE Access, vol. 4, pp. 774-791, 2016.

[13] D. Astely, E. Dahlman, G. Fodor, S. Parkvall, and J. Sachs, "LTE release 12 and beyond," IEEE Commun. Mag., vol. 51, no. 7, pp. 154-160, Jul. 2013.

[14] G. Zhao, S. Chen, L. Zhao and L. Hanzo, "A tele-traffic-aware optimal base-station deployment strategy for energy-efficient large-scale cellular networks," IEEE Access, vol. 4, pp. 2083-2095, 2016.

[15] K. Son, H. Kim, Y. Yi, and B. Krishnamachari, "Base station operation and user association mechanisms for energy-delay tradeoffs in green cellular networks," IEEE J. Sel. Areas Commun., vol. 29, no. 8, pp. 15251536, Sep. 2011. 
[16] L. Saker, S. E. Elayoubi, R. Combes, and T. Chahed, "Optimal control of wake up mechanisms of femtocells in heterogeneous networks," IEEE J. Sel. Areas Commun., vol. 30, no. 3, pp. 664-672, Apr. 2012.

[17] Z. Yang and Z. Niu, "Energy saving in cellular networks by dynamic RSBS association and BS switching," IEEE Trans. Veh. Technol., vol. 62, no. 9, pp. 4602-4614, Nov. 2013.

[18] L. Zhao, G. Zhao, and T. O'Farrell, "Efficiency metrics for wireless communications," in Proc. PIMRC 2013 (London, UK), Sept. 8-11, 2013, pp. 2825-2829.

[19] M. M. A. Hossain, K. Koufos, and R. Jäntti, "Energy efficient deployment of HetNets: impact of power amplifier and delay," in Proc. WCNC 2013 (Shanghai, China), Apr. 7-10, 2013, pp. 778-782.

[20] W. Guo and T. O'Farrell, "Dynamic cell expansion with self-organizing cooperation," IEEE J. Sel. Areas Commun., vol. 31, no. 5, pp. 851-860, May 2013

[21] H. Claussen, L. T. W. Ho, and F. Pivit, "Effects of joint macrocell and residential picocell deployment on the network energy efficiency," in Proc. PIMRC 2008 (Cannes, France), Sep. 15-18, 2008, pp. 1-6.

[22] F. Richter, A. J. Fehske, and G. P. Fettweis, "Energy efficiency aspects of base station deployment strategies for cellular networks," in (Proc. VTC 2009-Fall (Anchorage, AK), Sep. 20-23, 2009, pp. 1-5.

[23] J. G. Andrews, F. Baccelli, and R. K. Ganti, "A tractable approach to coverage and rate in cellular networks," IEEE Trans. Commun., vol. 59, no. 11, pp. 3122-3134, Nov. 2011.

[24] H. Zhang, S. Chen, L. Feng, Y. Xie, and L. Hanzo, "A universal approach to coverage probability and throughput analysis for cellular networks," IEEE Trans. Veh. Technol., vol. 64, no. 9, pp. 4245-4256, Sep. 2015.

[25] H. S. Dhillon, R. K. Ganti, F. Baccelli, and J. G. Andrews, "Modeling and analysis of K-tier downlink heterogeneous cellular networks," IEEE J. Sel. Areas Commun., vol. 30, no. 3, pp. 550-560, Apr. 2012.

[26] H. S. Jo, Y. J. Sang, P. Xia and J. G. Andrews, "Heterogeneous cellular networks with flexible cell association: A comprehensive downlink SINR analysis," IEEE Trans. Wireless Commun., vol. 11, no. 10, pp. 3484 3495, October 2012.

[27] S. Singh, H. S. Dhillon and J. G. Andrews, "Offloading in heterogeneous networks: Modeling, analysis, and design insights," IEEE Trans. Wireless Commun., vol. 12, no. 5, pp. 2484-2497, May 2013.

[28] X. Ge, J. Ye, Y. Yang, and Q. Li, "User mobility evaluation for 5G small cell networks based on individual mobility model," IEEE J. Sel. Area Commun., vol. 34, no. 3, pp. 528-541, Mar. 2016.

[29] J. Peng, P. Hong, and K. Xue, "Energy-aware cellular deployment strategy under coverage performance constraints," IEEE Trans. Wireless Commun., vol. 14, no. 1, pp. 69-80, Jan. 2015.

[30] S.-R. Cho and W. Choi, "Energy-efficient repulsive cell activation for heterogeneous cellular networks," IEEE J. Sel. Areas Commun., vol. 31, no. 5, pp. 870-882, May 2013

[31] Y. Huang, et al., "Energy-efficient design in heterogeneous cellular networks based on large-scale user behavior constraints," IEEE Trans. Wireless Commun., vol. 13, no. 9, pp. 4746-4757, Sep. 2014.

[32] Y. S. Soh, T. Q. S. Quek, M. Kountouris, and H. Shin, "Energy efficient heterogeneous cellular networks," IEEE J. Sel. Areas Commun., vol. 31 , no. 5, pp. 840-850, May 2013.

[33] X. Ge, et al., "Spatial spectrum and energy efficiency of random cellular networks," IEEE Trans. Commun., vol. 63, no. 3, pp. 1019-1030, Mar. 2015.

[34] G. Grimmett and D. Stirzaker, Probability and Random Processes (Third Edition). Cambridge University Press: Oxford, 2004.

[35] S. Srinivasa, Modeling Interference in Uniformly Random Wireless Networks: Theory and Applications. MSc Thesis, University of Notre Dame, USA, 2007.

[36] R. L. Streit, Poisson Point Processes: Imaging, Tracking and Sensing. London: Springer, 2010.

[37] J. Ferenc and Z. Néda, "On the size distribution of Poisson Voronoi cells," Physica A: Statistical Mechanics and its Applications, vol. 385, no. 2, pp. 518-526, 2007.

[38] S. Lee and K. Huang, "Coverage and economy of cellular networks with many base stations," IEEE Commun. Letters, vol. 16, no. 7, pp. 10381040, Jul. 2012.

[39] W. Guo, et al., "Automated small-cell deployment for heterogeneous cellular networks," IEEE Commun. Mag., vol. 51, no. 5, pp. 46-53, May 2013.

[40] Y. Zhou, et al., "Large-scale spatial distribution identification of base stations in cellular networks," IEEE Access, vol. 3, pp. 2987-2999, 2015.

[41] S. Tombaz, A. Vastberg, and J. Zander, "Energy- and cost-efficient ultrahigh-capacity wireless access," IEEE Wireless Commun., vol. 18, no. 5, pp. $18-24$, Oct. 2011
[42] Z. Hasan, H. Boostanimehr, and V. K. Bhargava, "Green cellular networks: a survey, some research issues and challenges," IEEE Commun. Surveys Tuts., vol. 13, no. 4, pp. 524-540, 4th quarter 2011.

[43] V. Mancuso and S. Alouf, "Reducing costs and pollution in cellular networks," IEEE Commun. Mag., vol. 49, no. 8, pp. 63-71, Aug. 2011.

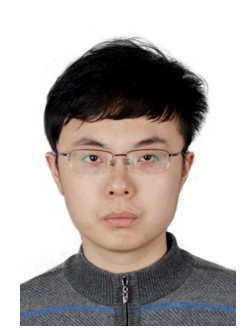

Guogang Zhao received the B.S. degree in communications engineering from People's Liberation Army Information Engineering University, Zhengzhou, Henan, China. He is currently working towards a Ph.D. degree at Department of Telecommunication Engineering, Xidian University, China. Since 2015, he has been a visiting Ph.D. student with Professor Hanzo and Professor Chen at School of Electronics and Computer Science, University of Southampton, Southampton, UK. His research interests focus on 5G wireless communication, network planning, ultra-dense cellular network.

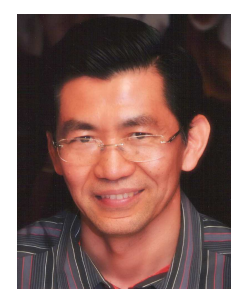

Sheng Chen (M'90-SM'97-F'08) received his BEng degree from the East China Petroleum Institute, Dongying, China, in 1982, and his $\mathrm{PhD}$ degree from the City University, London, in 1986, both in control engineering. In 2005, he was awarded the higher doctoral degree, Doctor of Sciences (DSc), from the University of Southampton, Southampton, UK.

From 1986 to 1999, He held research and academic appointments at the Universities of Sheffield, Edinburgh and Portsmouth, all in UK. Since 1999, he has been with the School of Electronics and Computer Science, the University of Southampton, UK, where he holds the post of Professor in Intelligent Systems and Signal Processing. Dr Chen's research interests include adaptive signal processing, wireless communications, modelling and identification of nonlinear systems, neural network and machine learning, intelligent control system design, evolutionary computation methods and optimisation. He has published over 550 research papers.

Dr. Chen is a Fellow of the United Kingdom Royal Academy of Engineering, a Fellow of IET, a Distinguished Adjunct Professor at King Abdulaziz University, Jeddah, Saudi Arabia, and an ISI highly cited researcher in engineering (March 2004). 


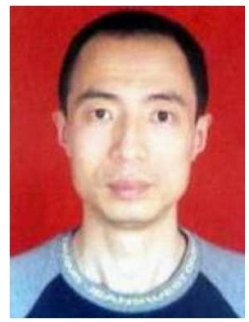

Liqiang Zhao obtained the BEng degree in Electrical Engineering from Shanghai Jiaotong University, China, in 1992, the MSc degree in Communications and Information Systems and the $\mathrm{PhD}$ degree in Information and Communications Engineering from Xidian University, China, in 2000 and 2003, respectively. In 2008, he was awarded by the Program for New Century Excellent Talents in University, Ministry of Education, China.

From 1992 to 2005, Dr. Zhao was a Senior Research Engineer with the 20th Research Institute, Chinese Electronics Technology Group Corporation, China, where his research focused on mobile communication systems and spread spectrum communications. From 2005 to 2007, he was an Associate Professor with State Key Laboratory of Integrated Service Networks, Xidian University, China, where his research focused on WiMAX, WLAN, and wireless sensor network. He was appointed as a Marie Curie Research Fellow at the Centre for Wireless Network Design, University of Bedfordshire in June 2007 to conduct research in the GAWIND project funded under EU FP6 HRM programme, where his activities focused on the area of automatic wireless broadband access network planning and optimization. Since June 2008, Professor Zhao has been with Xidian University. His current research focuses on broadband wireless communications and space communications. He has more than 70 publications in authorized academic periodicals both in China and abroad and in international science conferences, wherein 20 of which are retrieved in SCI, and more than 50 of them are EI indexed. He has also applied for 5 national invention patents. Professor Zhao has hosted/participated many vertical research projects, such as the National Natural Science Foundation, 863 Program and the National Science and Technology Major Projects, China, as well as many horizontal scientific research projects, including the EU FP6, FP7 Plans for International Cooperation and Exchange Projects, and the Huawei Fund.

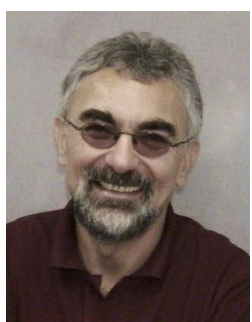

Lajos Hanzo FREng, FIEEE, FIET, Fellow of EURASIP, received the D.Sc. degree in electronics in 1976, the Ph.D. degree in 1983. In 2009, he was awarded the honorary doctorate, Doctor Honoris Causa, by the Technical University of Budapest.

During his 38-year career in telecommunications he has held various research and academic posts in Hungary, Germany and the UK. Since 1986 he has been with the School of Electronics and Computer Science, University of Southampton, UK, where he holds the chair in telecommunications. He has successfully supervised about $112 \mathrm{PhD}$ students, co-authored 18 John Wiley/IEEE Press books on mobile radio communications totalling in excess of 10000 pages, published 1703 research contributions at IEEE Xplore, acted both as TPC and General Chair of IEEE conferences, presented keynote lectures and has been awarded a number of distinctions. Currently he is directing a 50-strong academic research team, working on a range of research projects in the field of wireless multimedia communications sponsored by industry, the Engineering and Physical Sciences Research Council, UK, the European Research Council's Advanced Fellow Grant and the Royal Society's Wolfson Research Merit Award. He is an enthusiastic supporter of industrial and academic liaison and he offers a range of industrial courses. He is also a Governor of the IEEE VTS. From 2008 to 2012, he was the Editor-in-Chief of the IEEE Press and a Chaired Professor at Tsinghua University, China. 